\title{
Shells of selfdual lattices viewed as spherical designs
}

\author{
Claude PACHE \\ University of Geneva (Switzerland) \\ email: Claude.Pache@math. unige.ch
}

27 May 2004

\begin{abstract}
We find out for which $t$ shells of selfdual lattices and of theirs shadows are spherical $t$-designs. The method uses theta series of lattices, which are modular forms. We analyse fully cubic and Witt lattices, as well as all selfdual lattices of rank at most 24 .
\end{abstract}

\section{Introduction}

A nonempty finite subset of a Euclidean sphere provides approximations for integrals of functions defined on that sphere. In this context, such a subset is called a spherical design and its efficiency is measured by an integer $t \geqslant 0$ called its strength DeGoSe77 (precise definitions are given in Section 10. We are interested here in computing (or at least estimating) the strengths of shells in some selfdual lattices. These problems have natural formulations in terms of vanishing Fourier coefficients of modular forms which are appropriate theta series of the lattices.

The method used in this article was already used for finding the spherical design strengths of shells of extremal (even) lattices. (Do not confuse with "extreme lattice". The definition of extremal lattices of level 1 is given at the end of Section 5 ) See Venk84 and VenMar01 §16] for unimodular case, and BacVen01 for some other cases.

Let $\Lambda$ be a lattice in the standard Euclidian space $\mathbb{R}^{n}$; we denote by $\langle x \mid y\rangle$ the scalar product of two vectors $x, y \in \mathbb{R}^{n}$. For a positive number $m$, we denote by

$$
\Lambda_{m}:=\{\lambda \in \Lambda \mid\langle\lambda \mid \lambda\rangle=m\}
$$

the shell (or layer) of norm $m$ (that is to say of radius $\sqrt{m}$ in the usual sense of Euclidean geometry).

Given a lattice and a positive integer $t$, we would like to single out the three following questions:

(1) Is the shell of minimal norm a spherical $t$-design?

(2) Is some shell a spherical $t$-design?

(3) Is every shell a spherical $t$-design?

It is quite easy to show that, if any of these question is true for $t=2$ then the lattice is rational, that is proportional to an integral lattice (see, e.g., MartV01, Chap. $3, \S 1]$ ). It is therefore reasonable to restrict the discussion to integral lattices. 
Question (1) with $t=4$ (or $t=5$ which is equivalent in this case) asks whether a lattice is strongly perfect, which is the basic question in VenMar01. It is motivated by the classical result of Voronoi characterising extreme lattices as those which are "eutactic and perfect" Voro08. (A lattice is extreme if the density of the corresponding sphere packing of $\mathbb{R}^{n}$ is a local maximum in the space of all lattices.) Strongly perfect lattices in dimensions $n \leqslant 11$ have been classified (see VenMar01 for dimensions $n \leqslant 9$ and $n=11$, and NebVen00 for $n=10$ ); there are ten isometry classes, usually denoted by $\mathbf{Z}, \mathbf{A}_{2}, \mathbf{D}_{4}, \mathbf{E}_{6}, \mathbf{E}_{6}^{*}, \mathbf{E}_{7}, \mathbf{E}_{7}^{*}, \mathbf{E}_{8}, K_{10}^{\prime}$, and $K_{10}^{\prime}{ }^{*}$ (subscripts indicate dimensions).

Whenever Question (2) has a positive answer, it is an experimental fact that there exists a "rather small" $m$ for which $\Lambda_{m}$ is a spherical $t$-design, but we do not know any general result in this direction.

For the answer to Question (3) to be positive, it is sufficient that the space $\mathcal{H}^{(2 j)}\left(\mathbb{R}^{n}\right)^{\operatorname{Aut}(\Lambda)}$ of Aut $(\Lambda)$-invariant harmonic polynomials on $\mathbb{R}^{n}$ which are homogeneous of degree $2 j$ is reduced to $\{0\}$ for every positive integer $2 j \leqslant t$ (see GoeSei81 Thm. 3.12]).

Questions (1) to (3) make sense for various sets associated to a lattice $\Lambda$, and in particular for shadows of selfdual lattices. Recall that, if $\Lambda$ is an odd selfdual lattice with even part $\Lambda_{0}=\{\lambda \in \Lambda \mid\langle\lambda \mid \lambda\rangle \equiv 0 \bmod 2\}$, its shadow $\mathcal{S h}(\Lambda)$ is the complement of $\Lambda$ in the dual $\Lambda_{0}^{\sharp}$ of $\Lambda_{0}$. (Shadows enter naturally the discussion since they provide efficient tools to compute theta series.)

We denote by $\mathbf{Z}^{n}$ the cubic lattice of rank $n$. We denote by $\Gamma_{n}$ the Witt lattice of rank $n$, where $n$ is a multiple of 4 (see Section 10 for the definition); $\Gamma_{8}$ is the unique even selfdual lattice of rank 8 (also known as the Korkine-Zolotareff lattice). If $R$ is a root system of norm 2 , we denote by $R^{+}$a selfdual lattice of minimal norm 2 with $\Lambda_{2}=R$; it happens that, up to rank 23 , such a lattice is unique (whenever it exists). We denote by $k_{1} R_{1}+\cdots+k_{s} R_{s}$ the root system whose irreducible components are $R_{1}, \ldots, R_{s}$ with multiplicity $k_{1}, \ldots, k_{s}$ respectively; such a root system is called strongly eutactic if all its components have the same Coxeter number. Recall that the Leech lattice is the unique even selfdual lattice of rank 24 with minimal norm 4, and the shorter Leech lattice is the unique selfdual lattice of rank 23 with minimal norm 3. We say that a noncubic selfdual lattice $\Lambda$ of rank $n$ has a long shadow if $\sigma(\Lambda)=n-8$, where $\sigma(\Lambda)$ is the minimum norm of a characteristic (or parity) vector of $\Lambda$. The definitions of spherical $t$-design and $t^{1} / 2^{-}$-design are given in Section 1

Theorem A below is partly a reformulation of known results: Claims (i) to (iii) and some cases of Claims (iv) and (v) follow from results on harmonic polynomials that are invariant by the action of the automorphism group of the lattice, as explained above (GoeSei81, Thm. 3.2]; GoeSei79, Examples 7.6 and 7.7]).

\section{Theorem A.}

(i) All shells of the Leech lattice are spherical 11/1/2-designs.

(ii) All shells of the shorter Leech lattice and of its shadow are spherical 7-designs.

(iii) All shells of the Korkine-Zolotareff lattice are spherical $7 \frac{1}{2} 2^{\text {-designs. }}$

(iv) The following special shells of selfdual lattices of rank at most 24 and of their shadows are spherical 5-designs:

$$
\begin{array}{ll}
\left(\mathbf{Z}^{4}\right)_{m} & \text { for } m=2 a, \\
\left(\mathbf{Z}^{7}\right)_{m} & \text { for } m=4^{a}(8 b+3), a, b \geqslant 0, \\
\left(\mathcal{S h}\left(\mathbf{Z}^{16}\right)\right)_{m} & \text { for } m=4 a+2, a \geqslant 1,
\end{array}
$$




$$
\begin{array}{ll}
\left(4 \mathbf{A}_{5}\right)_{m}^{+},\left(5 \mathbf{D}_{4}\right)_{m}^{+} & \text {for } m=4^{a}, a \geqslant 0, \\
\left(2 \mathbf{D}_{8}\right)_{m}^{+} & \text {for } m=4 a+2, a \geqslant 1 .
\end{array}
$$

(v) All shells of the following lattices and of their shadows are spherical 3-designs: the cubic lattices $\mathbf{Z}^{n}$, the Witt lattices $\Gamma_{n}$, all selfdual lattices of rank at most 24, of minimum 2, and with strongly eutactic root system (see Definition 20. that includes all selfdual lattices of minimum 2 with long shadow and all even selfdual lattices of rank at most 24). Moreover some other selfdual lattices of rank at most 24 have some shells which are spherical 3-designs.

Our approach provides a setting for numerical computations; so we have checked that no shell of norm at most 1200 of selfdual lattices up to rank 24 or of theirs shadows is a spherical $t$-design for larger values of $t$ than those indicated in the theorem.

See Theorems [25, 28, 32, 36, 38, and 43 for details.

Let

$$
\Delta_{24}=q^{2} \prod_{m \geqslant 1}\left(1-q^{2 m}\right)^{24}=\sum_{m \geqslant 1} \tau(m) q^{2 m}
$$

be the generating series of the Ramanujan numbers $\tau(m)$. It is a famous conjecture of Lehmer that $\tau(m)$ is never zero, and it has been verified for $m \leqslant 10^{15}$ Serr85, $\S 3.3]$. The following Proposition gives a reformulation of that conjecture in terms of spherical design strengths of shells of the Korkine-Zolotareff lattice and of the even selfdual lattices of rank 16. It is Proposition 33 in our article.

Proposition B. For $m \geqslant 1$, the following are equivalent:

(a) $\tau(m)=0$;

(b) the shell of norm $2 m$ of the Korkine-Zolotareff lattice is an 8-design (and therefore an 11-design);

(c) the shell of norm $2 m$ of any even selfdual lattice $\Lambda$ of rank 16, is a 4-design (and therefore a 7-design);

[Note that, for example, in Condition (c) above, the shells of $\Lambda$ are not only


4-design, then it is a 7-design.]

Here are other similar equivalences between spherical design strengths of shells of lattices and vanishing Fourier coefficients of modular forms. (The definitions of the series $\Delta_{8}, \theta_{2}, \theta_{3}$, and $\theta_{4}$ appear in Section 5 )

Proposition C. Consider any of the following choice of a selfdual lattice $\Lambda$, a positive integer $t$, and a series $\Theta(z)=\sum_{m \geqslant 1} a_{m} q^{m}$ where $q=e^{i \pi z}$.

$-\Lambda=\mathbf{Z}^{n}$ the cubic lattice of rank $n \geqslant 2, t=3, \Theta=\Delta_{8} \theta_{3}^{n}$ (Section Q);

$-\Lambda=\Gamma_{n}$ the Witt lattice of rank $n \geqslant 12, n \equiv 0 \bmod 4, t=3, \Theta=\theta_{2}^{4} \theta_{3}^{4} \theta_{4}^{4} \times$ $\left(-\theta_{2}^{n-4}+\theta_{3}^{n-4}-\theta_{4}^{n-4}\right)$ (Section [10);

- $\Lambda$ the Korkine-Zolotareff lattice, of rank $8, t=7, \Theta=\Delta_{24}$ (Section [11);

$-\Lambda$ an even selfdual lattice of rank $16, t=3, \Theta=\Delta_{24}$ (Section [11);

- $\Lambda$ one of the 23 Niemeier lattice, of rank $24, t=3, \Theta=\mathrm{Q} \Delta_{24}$ (Section [11);

- $\Lambda$ the Leech lattice, $t=11, \Theta=\Delta_{24}^{2}$ (Section 11); 
- $\Lambda$ one of the 12 odd selfdual lattices with long shadow and of minimum 2 (with rank $n=12$ or $14 \leqslant n \leqslant 22$ ), $t=3, \Theta=\Delta_{8}^{2} \theta_{3}^{n-8}$ (Section [12);

- $\Lambda$ the shorter Leech lattice, of rank 23, $t=7, \Theta=\Delta_{8}^{2} \theta_{3}^{15}$ (Section [12).

Then, for each $m \geqslant 1$, the shell of $\Lambda$ of norm $m$ is a spherical $t$-design; moreover it is not a spherical $(t+1)$-design if and only if $a_{m} \neq 0$.

It is therefore interesting to look for vanishing coefficients of the series mentioned above. For example, we ask the following questions:

(1) Consider the series

$$
\Delta_{24}^{2}=\sum_{m \geqslant 2} a_{m} q^{2 m}, \quad a_{m}=\sum_{i+j=m} \tau(i) \tau(j),
$$

where $\tau(i)$ is the $i$ th Ramanujan number. Is it true that $a_{m} \neq 0$ for every $m \geqslant 2$ ?

(2) Consider the series

$$
\Delta_{8} \theta_{3}^{n}=\sum_{m \geqslant 1} a_{m} q^{m}
$$

Is it true that $a_{m} \neq 0$ for every positive integer $m$ not of the form $4^{a}(8 b+3)$, $a, b \geqslant 0$ ?

(There are similar questions for the other forms mentioned in Proposition C.)

We have checked numerically that the answers are positive for $m \leqslant 1200$.

In Sections 1 to 5 we recall standard material on spherical designs, selfdual lattices, theta series, and modular forms. Section 6 gives the form of the theta series of selfdual lattices; this is the centre of our analysis. Some indices of vanishing Fourier coefficients for modular forms are given in Section 7 In Section 8 we analyse the spherical design strengths of root systems of norm 2. Sections 9 to 14 contain the results on the strength of shells of some selfdual lattices, namely the two infinite series of cubic and Witt lattices, and all selfdual lattices of rank at most 24. Finally, an appendix contains an alternative proof not using modular forms of the fact that appropriate shells of $\mathbf{Z}^{4}$ and $\mathbf{Z}^{7}$ are spherical 5-designs. 


\section{A General theory}

\section{Spherical designs}

Let $n \geqslant 2$ be an integer, and let $m$ be a positive real number; we denote by

$$
\mathbb{S}_{m}^{n-1}:=\left\{x \in \mathbb{R}^{n} \mid\langle x \mid x\rangle=m\right\}
$$

the sphere of square radius $m$, and by $\sigma$ the probability measure on $\mathbb{S}_{m}^{n-1}$ invariant under the action of the orthogonal group $\mathrm{O}(n)$. A spherical design of strength $t$, or a $t$-spherical design, is a nonempty finite subset $X \subseteq \mathbb{S}_{m}^{n-1}$ such that

$$
\frac{1}{|X|} \sum_{x \in X} P(x)=\int_{\mathbb{S}_{m}^{n-1}} P(y) d \sigma(y)
$$

for every polynomial form $P$ on $\mathbb{R}^{n}$ of degree at most $t$ DeGoSe77.

We denote by $\mathcal{H}^{(j)}\left(\mathbb{R}^{n}\right)$ the set of homogeneous polynomial forms on $\mathbb{R}^{n}$ of degree $j$ that are harmonic. It is classical that $X$ is a spherical $t$-design if and only if the condition

$$
\sum_{x \in X} P(x)=0, \quad \forall P \in \mathcal{H}^{(j)}\left(\mathbb{R}^{n}\right)
$$

holds for every integer $j$ such that $1 \leqslant j \leqslant t$. It is indeed an immediate consequence of the decomposition

$$
\mathcal{P}^{(t)}\left(\mathbb{R}^{n}\right)=\mathcal{H}^{(t)}\left(\mathbb{R}^{n}\right) \oplus \omega \mathcal{P}^{(t-2)}\left(\mathbb{R}^{n}\right),
$$

where $\mathcal{P}^{(t)}\left(\mathbb{R}^{n}\right)$ is the space of homogeneous polynomial forms on $\mathbb{R}^{n}$ of degree $t$, and $\omega(x):=\langle x \mid x\rangle$; see Vile68, §IX.2] and MartV01, Chap. 1, §§2,3].

In this article, we study spherical designs which are shells of selfdual lattices or of their shadows. These designs are antipodal sets, that is sets $X$ satisfying $-X=X$; in this case, Condition $\left(C_{j}\right)$ is automatically fulfilled for $j$ odd. Therefore we use the following reformulation for antipodal sets:

1. Definition. A nonempty finite antipodal subset $X \subseteq \mathbb{S}_{m}^{n-1}$ is a spherical $(2 s+1)$-design (or, equivalently, a spherical $2 s$-design) if the condition

$$
\sum_{x \in X} P(x)=0, \quad \forall P \in \mathcal{H}^{(2 j)}\left(\mathbb{R}^{n}\right)
$$

holds for every even integer $2 j$ such that $2 \leqslant 2 j \leqslant 2 s$.

Some antipodal spherical $(2 s+1)$-designs, although not satisfying Condition $\left(C_{2 s+2}\right)$, do verify Condition $\left(C_{2 s+4}\right)$. Therefore, we define Venk84:

2. Definition. A nonempty finite antipodal subset $X \subseteq \mathbb{S}_{m}^{n-1}$ is a spherical $\left(2 s+1+\frac{1}{2}\right)$-design if it verifies condition $\left(C_{2 j}\right)$ for $2 \leqslant 2 j \leqslant 2 s$ and $2 j=2 s+4$.

\section{Selfdual lattices and shadows}

For a subset $A \subseteq \mathbb{R}^{n}$ and a positive real number $m>0$, the shell (or layer) of norm $m$ of $A$ is

$$
A_{m}:=\{x \in \Lambda \mid\langle x \mid x\rangle=m\}=A \cap \mathbb{S}_{m}^{n-1} .
$$

A lattice of rank $n$ is a discrete $\mathbb{Z}$-submodule of $\mathbb{R}^{n}$ which spans $\mathbb{R}^{n}$ as $\mathbb{R}$-module. Two lattices are equivalent if there exists an orthogonal linear transformation which 
sends one lattice onto the other; we often consider two equivalent lattices as being the same lattice. We define the minimum (or the minimal norm) of $\Lambda$ as

$$
\min (\Lambda):=\min \left\{m>0 \mid \Lambda_{m} \neq \emptyset\right\} .
$$

The dual of a lattice $\Lambda$ is the lattice

$$
\Lambda^{\sharp}:=\left\{y \in \mathbb{R}^{n} \mid\langle y \mid x\rangle \in \mathbb{Z} \forall x \in \Lambda\right\} .
$$

The lattice $\Lambda$ is called integral if $\Lambda \subseteq \Lambda^{\sharp}$, that is if $\langle x \mid y\rangle \in \mathbb{Z}$ for all $x, y \in \Lambda$. An integral lattice $\Lambda$ is called even if $\langle x \mid x\rangle \in 2 \mathbb{Z}$ for all $x \in \Lambda$; it is called odd otherwise. An integral lattice is called selfdual (or unimodular) if $\Lambda^{\sharp}=\Lambda$.

For $A \subseteq \mathbb{R}^{a}$ and $B \subseteq \mathbb{R}^{b}$ we set $A \oplus B:=\left\{(x, y) \in \mathbb{R}^{a+b} \mid x \in A, y \in B\right\}$. It is easily checked that any integral lattice $\Lambda$ of rank $n$ is of the form

$$
\Lambda \simeq \mathbf{Z}^{p} \oplus L,
$$

where $L$ is an integral lattice of rank $N=n-p$ and of minimum at least 2, and where

$$
\mathbf{Z}^{p}:=\left\{\left(x_{1}, \ldots, x_{p}\right) \mid x_{i} \in \mathbb{Z}\right\} \subseteq \mathbb{R}^{p}
$$

is the cubic lattice of rank $p$. Note that $L$ is selfdual if and only if $\Lambda$ is selfdual.

The shadow $\operatorname{Sh}(\Lambda)$ of a selfdual lattice $\Lambda$ is defined as follows: If $\Lambda$ is even, we set $\operatorname{Sh}(\Lambda):=\Lambda$. Otherwise let

$$
\Lambda_{0}:=\{x \in \Lambda \mid\langle x \mid x\rangle \equiv 0 \bmod 2\},
$$

which is an even sublattice of $\Lambda$ of index 2 ; therefore $\Lambda$ is a sublattice of $\Lambda_{0}^{\sharp}$ of index 2 . We set

$$
\operatorname{Sh}(\Lambda):=\Lambda_{0}^{\sharp} \backslash \Lambda \text {. }
$$

An alternative description of the shadow is $\mathcal{S h}(\Lambda)=\{x / 2 \mid x$ is a characteristic vector of $\Lambda$, where a characteristic vector (or parity vector) of $\Lambda$ is a vector $x \in \Lambda$ such that $\langle x \mid y\rangle \equiv\langle y \mid y\rangle \bmod 2$ for all $y \in \Lambda$.

For a selfdual lattice $\Lambda$, we define:

$$
\sigma(\Lambda):=4 \min \{\langle x \mid x\rangle \mid x \in \mathcal{S h}(\Lambda)\},
$$

which is a nonnegative integer. (It is the minimal norm of the characteristic vectors of $\Lambda$.) We have $\sigma(\Lambda)=0$ if and only if $\Lambda$ is even.

It is easily checked that, if $\Lambda^{\prime}$ and $\Lambda^{\prime \prime}$ are selfdual lattices, then $\Lambda^{\prime} \oplus \Lambda^{\prime \prime}$ is a selfdual lattice, and

$$
\begin{gathered}
\mathcal{S h}\left(\Lambda^{\prime} \oplus \Lambda^{\prime \prime}\right)=\operatorname{Sh}\left(\Lambda^{\prime}\right) \oplus \mathcal{S h}\left(\Lambda^{\prime \prime}\right), \\
\sigma\left(\Lambda^{\prime} \oplus \Lambda^{\prime \prime}\right)=\sigma\left(\Lambda^{\prime}\right)+\sigma\left(\Lambda^{\prime \prime}\right) .
\end{gathered}
$$

The following facts are well-known; a proof using modular forms appears at the end of Section 5

3. Proposition. Let $\Lambda \subseteq \mathbb{R}^{n}$ be a selfdual lattice. Then

(i) for every $x \in \mathcal{S h}(\Lambda)$, we have $4\langle x \mid x\rangle \equiv n \bmod 8$;

(ii) there exists a nonnegative integer $k$ such shat $\sigma(\Lambda)=n-8 k$. In particular, if $\Lambda$ is even, then $n \equiv 0 \bmod 8$;

(iii) we have $\sigma(\Lambda)=n$ if and only if $\Lambda \simeq \mathbf{Z}^{n}$,

The characterisation of $\mathbf{Z}^{n}$ given in Claim (iii) is due to Elkies Elki95a].

Note that, in the decomposition $\Lambda \simeq \mathbf{Z}^{p} \oplus L$, if $\sigma(\Lambda)=n-8 k$, then $\sigma(L)=$ $N-8 k$, where $n$ is the rank of $\Lambda$, and $N$ is the rank of $L$.

The list of selfdual lattices of rank at most 24 can be found in ConSlo99, Chap. 16 and 17] and [Bach97. 


\section{Theta series}

Let us now introduce the tools for analysing the spherical design strengths of shells of selfdual lattices. Let

$$
\mathbb{H}:=\{z \in \mathbb{C} \mid \Im z>0\}
$$

be the Poincaré half-plane. Recall that a holomorphic function $f: \mathbb{H} \rightarrow \mathbb{C}$ is bounded at infinity if there exists $r>0$ such that $\{f(z) \mid \Im z \geqslant r\}$ is bounded.

4. Lemma - Definition. Let $A$ be a nonempty subset of $\mathbb{R}^{n}$ for which there exists $\delta>0$ such that $|x-y| \geqslant \delta$ for all distinct $x, y \in A$. Let $P$ be a polynomial form on $\mathbb{R}^{n}$. Then the series

$$
\Theta_{A, P}(z):=\sum_{x \in A} P(x) e^{i \pi z\langle x \mid x\rangle}, \quad z \in \mathbb{H},
$$

converges absolutely to a function on $\mathbb{H}$ that is holomorphic and bounded at infinity. It is the theta series of $A$ weighted by $P$.

Let us assume moreover that there exists a real number $\alpha>0$ such that $\alpha\langle x \mid x\rangle \in 2 \mathbb{Z}$ for every $x \in A$. Then we have

$$
\Theta_{A, P}(z+\alpha)=\Theta_{A, P}(z), \quad \forall z \in \mathbb{H} .
$$

Proof. The condition on the distance of two distinct points of $A$ implies that there exist a constant $C>0$ such that for every $r \geqslant 0$ the set

$$
\{x \in A \mid r \leqslant\langle x \mid x\rangle \leqslant r+1\}
$$

contains at most $C r^{n-1}$ points; the absolute convergence of $\Theta_{A, P}$ follows. The second claim of the lemma is straightforward.

Remarks.

(i) For a holomorphic function $F: \mathbb{H} \rightarrow \mathbb{C}$ verifying $F(z+\alpha)=F(z)$ for all $z \in \mathbb{H}$ and for some $\alpha>0$, the condition to be bounded at infinity is equivalent to the condition that there exists a Fourier expansion of the form

$$
F(z)=\sum_{m \in 2 \alpha^{-1} \mathbb{N}} a_{m} e^{i \pi z m}
$$

which converges for $\Im z$ sufficiently large. (We use $\mathbb{N}=\{0,1,2, \ldots\}$.) In this case, $F$ is said to be holomorphic at infinity.

(ii) For a real number $m$ and if $z \in \mathbb{H}$ is understood, we write

$$
q^{m}:=e^{i \pi z m}, \quad \text { where } z \in \mathbb{H}
$$

Thus the theta series of $A$ weigted by $P$ can be written

$$
\Theta_{A, P}(z)=\sum_{x \in A} P(x) q^{\langle x \mid x\rangle}=\sum_{m \in 2 \alpha^{-1} \mathbb{N}} a_{m}^{(P)} q^{m}, \quad \text { where } a_{m}^{(P)}:=\sum_{x \in A_{m}} P(x) .
$$

(iii) The classical theta series of $A$ is

$$
\Theta_{A}:=\Theta_{A, 1}=\sum_{m \geqslant 0}\left|A_{m}\right| q^{m}
$$


(iv) Let $A \subseteq \mathbb{R}^{a}$ and $B \subseteq \mathbb{R}^{b}$, and let $P$, respectively $Q$, be polynomial forms on $\mathbb{R}^{a}$, respectively $\mathbb{R}^{b}$. Then

$$
\Theta_{A \oplus B, P Q}=\Theta_{A, P} \Theta_{B, Q} .
$$

Now we reformulate the condition of being a spherical design using theta series.

5. Lemma. Let $A$ be a nonempty subset of $\mathbb{R}^{n}$ such that there exist $\delta>0$ verifying $|x-y| \geqslant \delta$ for all distinct $x, y \in A$. Then, for $m>0$, the shell $A_{m}$ is a spherical $t$-design or is empty if and only if

$$
a_{m}^{(P)}=0 \text { for every } P \in \mathcal{H}^{(2 j)}\left(\mathbb{R}^{n}\right), 1 \leqslant 2 j \leqslant t,
$$

where $a_{m}^{(P)}$ are the Fourier coefficients of the theta series

$$
\Theta_{A, P}(z)=\sum_{m} a_{m}^{(P)} q^{m}
$$

Proof. This follows directly from the definitions. Note that the Fourier coefficients $a_{m}^{(P)}$ are retrieved from $\Theta_{A, P}$ by the formula

$$
a_{m}^{(P)}=\lim _{R \rightarrow \infty} \frac{1}{2 R} \int_{-R}^{R} e^{-i \pi m\left(x+i y_{0}\right)} \Theta_{A, P}\left(x+i y_{0}\right) d x,
$$

valid for any $y_{0}>0$.

\section{Modular forms}

The interest of theta series of lattices is that they have the following property, which will help to compute them, at least if the lattice is selfdual. This proposition is a direct consequence of the Poisson Summation Formula; see for example Ebel94. Prop. 3.1, p. 87].

6. Proposition. Let $\Lambda \subseteq \mathbb{R}^{n}$ be a lattice, and let $P: \mathbb{R}^{n} \rightarrow \mathbb{C}$ be a harmonic polynomial of degree $2 j$. Then

$$
\Theta_{\Lambda^{\sharp}, P}(z)=(\operatorname{det} \Lambda)^{1 / 2}(-1)^{j}(i / z)^{n / 2+2 j} \Theta_{\Lambda, P}(-1 / z) .
$$

(For the power of $i / z$, we use the principal branch; observe that $-\pi / 2 \leqslant \arg (i / z) \leqslant$ $\pi / 2$ for $z \in \mathbb{H}$.)

In the case of selfdual lattices, the latter formula gives a relation between $\Theta_{\Lambda, P}(z)$ and $\Theta_{\Lambda, P}(-1 / z)$. To be more precise, let us give the following definitions. Recall that $\mathbb{N}=\{0,1,2, \ldots\}$.

\section{Definition.}

(i) Let $\lambda \in\{1,2\}, \omega \in \frac{1}{2} \mathbb{N}$, and $\epsilon \in\{+,-\}$. A modular form of signature $(\lambda, \omega, \epsilon)$ is a holomorphic and holomorphic at infinity function $f: \mathbb{H} \rightarrow \mathbb{C}$ that verifies

$$
\begin{gathered}
f(z+\lambda)=f(z), \\
f(-1 / z)=\epsilon(z / i)^{\omega} f(z),
\end{gathered}
$$

for all $z \in \mathbb{H}$. The number $\omega$ is the weight of the form. We denote by $\mathcal{M}_{\omega}^{\lambda, \epsilon}$ the vector space of modular forms of signature $(\lambda, \omega, \epsilon)$; note that $\mathcal{M}_{\omega}^{1, \epsilon} \subseteq \mathcal{M}_{\omega}^{2, \epsilon}$. We set:

$$
\mathcal{M}^{\lambda, \epsilon}:=\bigoplus_{\omega \in(1 / 2) \mathbb{N}} \mathcal{M}_{\omega}^{\lambda, \epsilon}, \quad \mathcal{M}^{\lambda}:=\mathcal{M}^{\lambda,+} \oplus \mathcal{M}^{\lambda,-} .
$$

Observe that $\mathcal{M}^{\lambda}$ and $\mathcal{M}^{\lambda,+}$ are algebras, graded by the weight. 
(ii) A modular form $f$ is parabolic if

$$
\lim _{\Im z \rightarrow+\infty} f(z)=0 .
$$

Nonzero modular forms of signature $(1, \omega, \epsilon)$ exist only for weights $\omega \in 4 \mathbb{N}$ if $\epsilon=+$, and for weights $\omega \in 4 \mathbb{N}+2$ if $\epsilon=-$. Indeed, let $S: z \mapsto-1 / z$ and $T: z \mapsto z+1$. We have STSTST $=\operatorname{id}_{\mathbb{H}}$, therefore $f(S T S T S T z)=f(z)$ for every $z \in \mathbb{H}$. On the other hand, it is straightforward to check that, for $f \in \mathcal{M}_{\omega}^{1, \epsilon}$,

$$
f(\operatorname{STSTST} z)=\epsilon i^{\omega} f(z),
$$

where $i$ must be taken with argument $\pi / 2$. Thus $\epsilon e^{i \omega \pi / 2}=1$ if $f \neq 0$.

Nonzero modular forms of signature $(2, \omega, \epsilon)$ exist for every weight $\omega \in \frac{1}{2} \mathbb{N}$; for example, according to Proposition 8 below, $\theta_{3}^{n}=\Theta_{\mathbf{Z}^{n}}$ is a modular form of parameters $(1, n / 2,+)$.

Modular forms of signature $(1, \omega, \epsilon)$, are the classical modular forms for $\operatorname{SL}(2, \mathbb{Z})$. Modular forms of signature $(2, \omega, \epsilon)$ are modular forms for the subgroup of index 3 in $\operatorname{SL}(2, \mathbb{Z})$ whose elements are the matrices that reduce to $\left(\begin{array}{ll}1 & 0 \\ 0 & 1\end{array}\right)$ or $\left(\begin{array}{ll}0 & 1 \\ 1 & 0\end{array}\right)$ modulo 2 (sometimes noted $G(2)$ or $\Gamma_{V}(2)$ ).

Proposition [ implies immediately:

8. Proposition. Let $\Lambda \subseteq \mathbb{R}^{n}$ be a selfdual lattice, and let $P \in \mathcal{H}^{(2 j)}\left(\mathbb{R}^{n}\right)$. Then

$$
\begin{array}{ll}
\Theta_{\Lambda, P} \in \mathcal{M}_{n / 2+2 j}^{2,+} & \text { if } 2 j \equiv 0 \bmod 4, \\
\Theta_{\Lambda, P} \in \mathcal{M}_{n / 2+2 j}^{2,-} & \text { if } 2 j \equiv 2 \bmod 4 .
\end{array}
$$

If moreover $\Lambda$ is even, then

$$
\begin{array}{ll}
\Theta_{\Lambda, P} \in \mathcal{M}_{n / 2+2 j}^{1,+} & \text { if } 2 j \equiv 0 \bmod 4, \\
\Theta_{\Lambda, P} \in \mathcal{M}_{n / 2+2 j}^{1,-} & \text { if } 2 j \equiv 2 \bmod 4 .
\end{array}
$$

Moreover, if $2 j>0$ then $\Theta_{\Lambda, P}$ is parabolic.

Let us now look at theta series of shadows of selfdual lattices.

9. Definition. Let $f \in \mathcal{M}_{\omega}^{2, \epsilon}$. The shadow of $f$ is the function $\mathcal{S h} f: \mathbb{H} \rightarrow \mathbb{C}$ defined by

$$
\operatorname{Shf}(z):=(i / z)^{\omega} f(-1 / z+1) .
$$

10. Proposition. Let $\Lambda$ be a selfdual lattice, and let $P \in \mathcal{H}^{(2 j)}\left(\mathbb{R}^{n}\right)$. Then

$$
\Theta_{\mathcal{S h}(\Lambda), P}=(-1)^{j} \mathcal{S h} \Theta_{\Lambda, P} .
$$

Proof. If $\Lambda$ is even, this follows immediatly from $\operatorname{Sh}(\Lambda)=\Lambda$ and from Proposition 8 If $\Lambda$ is odd, we observe that

$$
2 \Theta_{\Lambda_{0}, P}(z)=\Theta_{\Lambda, P}(z)+\Theta_{\Lambda, P}(z+1)
$$

where $\Lambda_{0}$ is the even sublattice of $\Lambda$, of index 2. Then we apply Proposition [ 6 to $\Lambda_{0}$ and $\Lambda$ to obtain, for $P \in \mathcal{H}^{(2 j)}\left(\mathbb{R}^{n}\right)$,

$$
\begin{aligned}
\Theta_{\mathcal{S h}(\Lambda), P} & =\Theta_{\Lambda_{0}^{\sharp}, P}(z)-\Theta_{\Lambda, P}(z) \\
& =2(-1)^{j}(i / z)^{n / 2+2 j} \Theta_{\Lambda_{0}, P}(-1 / z)-(-1)^{j}(i / z)^{n / 2+2 j} \Theta_{\Lambda, P}(-1 / z) \\
& =(-1)^{j} \mathcal{S h} \Theta_{\Lambda, P}(z) .
\end{aligned}
$$




\section{A theorem of classification for modular forms}

In order to use Proposition 8 we need a description of the vector spaces $\mathcal{M}_{\omega}^{\lambda, \epsilon}$ defined in the previous section. There are very nice results of classification that we recall in this section.

Before giving examples of modular forms, it is convenient (although not essential) to formulate a definition of the weight which applies to a larger class of functions than modular forms:

11. Definition. Let $\omega \in \mathbb{R}$. Let $f$ and $g$ be two meromorphic functions on $\mathbb{H}$. Assume that there exist $\alpha$ and $\beta>0$ such that

$$
\begin{gathered}
f(z+\alpha)=f(z), \\
g(z+\beta)=g(z), \\
f(-1 / z)=(z / i)^{\omega} g(z),
\end{gathered}
$$

for all $z \in \mathbb{H}$. (Note that the last condition is symmetric in $f$ and $g$.) We say that $f$ and $g$ are of weight $\omega$.

When $f$ is a modular form of signature $(\lambda, \omega, \epsilon)$ (Definition [7), the assumptions of the Definition above are verified for $\alpha=\beta=\lambda$ and $g=\epsilon f$, and so $f$ is of weight $\omega$ as expected. However, some functions on $\mathbb{H}$ that are holomorphic and holomorphic at infinity, although not being modular forms, do have a weight in the sense of Definition [1] For example, we have $\theta_{4}(-1 / z)=(z / i)^{1 / 2} \theta_{2}(z)$, where $\theta_{4}$ and $\theta_{2}$ are the two periodic functions in $z$ defined below. Therefore $\theta_{2}$ and $\theta_{4}$ are both of weight $1 / 2$; but they are not modular forms.

It is easily checked that the algebra of meromorphic functions $f$ on $\mathbb{H}$ satisfying the assumptions of Definition [1] with some meromorphic function $g$ and some rational positive numbers $\alpha$ and $\beta$ is an algebra graded by the weight.

We give here a list of functions that have a weight in the sense of the Definition [11] Recall that we write $q^{m}:=e^{i \pi z m}$.

$$
\begin{array}{rlr}
\theta_{2}(z) & =\sum_{m \in \mathbb{Z}+1 / 2} q^{m^{2}}=2 q^{1 / 4}\left(1+q^{2}+O\left(q^{6}\right)\right) & \text { of weight } 1 / 2, \\
\theta_{3}(z)=\sum_{m \in \mathbb{Z}} q^{m^{2}}=1+2 q+2 q^{4}+O\left(q^{9}\right) & \text { of weight } 1 / 2, \\
\theta_{4}(z)=\sum_{m \in \mathbb{Z}}(-q)^{m^{2}}=1-2 q+2 q^{4}+O\left(q^{9}\right) & \text { of weight } 1 / 2, \\
\theta_{2}^{4}+\theta_{4}^{4}=\theta_{3}^{4} & \\
\Phi(z)=\theta_{4}^{4}(z)-\theta_{2}^{4}(z)=1-24 q+24 q^{2}-96 q^{3}+O\left(q^{4}\right) & \text { of weight 2, } \\
\Delta_{8}(z)=\frac{1}{16} \theta_{2}^{4}(z) \theta_{4}^{4}(z)=q-8 q^{2}+28 q^{3}+O\left(q^{4}\right) & \text { of weight } 4, \\
\mathrm{Q}(z)=\theta_{3}^{8}(z)-16 \Delta_{8}(z)=1+240 q^{2}+2160 q^{4}+O\left(q^{6}\right) & \text { of weight } 4, \\
\mathrm{R}(z)=\Phi(z)\left(\theta_{3}^{8}(z)+8 \Delta_{8}(z)\right)=1-504 q^{2}+O\left(q^{4}\right) & \text { of weight } 6, \\
\Delta_{24}(z)=\theta_{3}^{8}(z) \Delta_{8}^{2}(z)=q^{2}-24 q^{4}+252 q^{6}+O\left(q^{8}\right) & \text { of weight } 12 .
\end{array}
$$

The coefficients of the latter form

$$
\Delta_{24}(z)=\sum_{m \geqslant 1} \tau(m) q^{2 m}
$$


are the celebrated Ramanujan numbers. All the functions listed above can be expressed in terms of the Jacobi theta function

$$
\theta_{3}(\xi \mid z)=\sum_{m \in \mathbb{Z}} e^{2 i \xi m+i \pi z m^{2}}, \quad z \in \mathbb{H}
$$

indeed, we have

$$
\theta_{2}(z)=e^{i \pi z / 4} \theta_{3}(\pi z / 2 \mid z), \quad \theta_{3}(z)=\theta_{3}(0 \mid z), \quad \theta_{4}(z)=\theta_{3}(\pi / 2 \mid z) .
$$

Let $\mathcal{M}$ be the algebra generated by $\theta_{2}, \theta_{3}$ and $\theta_{4}$. It is an algebra graded by the weight, where the weight ranges over the set of nonnegative half-integer. The elements of weight zero are the constants.

The definition of the shadow of a modular form (Definition 9) carries over to functions having a weight in the sense of Definition 11] and provides an endomorphism of the graded algebra $\mathcal{M}$. We give here shadows of some functions in $\mathcal{M}$.

$$
\begin{array}{r}
\mathcal{S h} \theta_{2}=\sqrt{i} \theta_{4}, \quad \mathcal{S h} \theta_{4}=\theta_{3}, \quad \operatorname{Sh} \theta_{3}=\theta_{2}, \\
\mathcal{S h} \Delta_{8}(z)=-\frac{1}{16}+q^{2}-7 q^{4}+O\left(q^{6}\right), \\
\operatorname{Sh} \Phi(z)=2+48 q^{2}+48 q^{4}+O\left(q^{6}\right), \\
\mathcal{S h} \mathrm{Q}=\mathrm{Q}, \quad \operatorname{Sh} \mathrm{R}=-\mathrm{R}, \quad \mathcal{S h} \Delta_{24}=\Delta_{24} .
\end{array}
$$

These formulae (and many other useful identities) can be found in ConSlo99, Chap. $4, \S 4.1]$.

All modular forms of signature $(1, \omega, \pm)$ or $(2, \omega, \pm)$ are elements of $\mathcal{M}$. More precisely:

\section{THEOREM.}

$$
\begin{array}{lll}
\mathcal{M}^{2,+}=\mathbb{C}\left[\theta_{3}, \Delta_{8}\right], & & \mathcal{M}^{1,+}=\mathbb{C}\left[\mathrm{Q}, \Delta_{24}\right], \\
\mathcal{M}^{2,-}=\Phi \mathbb{C}\left[\theta_{3}, \Delta_{8}\right], & \mathcal{M}^{1,-}=\mathrm{R} \mathbb{C}\left[\mathrm{Q}, \Delta_{24}\right] .
\end{array}
$$

For a proof of this deep result, see [Rank77, Section 6.1 for $\mathcal{M}^{1, \pm}$, and Section 7.1 for $\mathcal{M}^{2, \pm}$. The notation of Rank77] are:

$\{\Gamma(1), \omega\}$ for $\mathcal{M}_{\omega}^{1,+}$ when $\omega \in 4 \mathbb{N}$, and for $\mathcal{M}_{\omega}^{1,-}$ when $\omega \in 4 \mathbb{N}+2$;

$\left\{\Gamma_{V}(2), \omega, v_{2 \omega}\right\}$ for $\mathcal{M}_{\omega}^{2,+}, \omega \in \frac{1}{2} \mathbb{N}$, and

$\left\{\Gamma_{V}(2), \omega, v_{2 \omega}^{*}\right\}$ for $\mathcal{M}_{\omega}^{2,-}, \omega \in \frac{1}{2} \mathbb{N}$.

Moreover modular forms are called there integral modular forms.

Here is a first application of this theorem:

Proof of Proposition 3. For brevity, we write $\Theta_{\Lambda}, \Delta_{8}$, etc. instead of $\Theta_{\Lambda}(z), \Delta_{8}(z)$, etc. By Theorem 12 and Proposition 8 for $\Lambda$ a selfdual lattice, the theta series

$$
\Theta_{\Lambda}=1+\sum_{m \geqslant 1}\left|\Lambda_{m}\right| q^{m}
$$

is a polynomial in $\theta_{3}$ and $\Delta_{8}$ and is of weight $n / 2$; therefore it is of the form

$$
\Theta_{\Lambda}=\theta_{3}^{n}+c_{1} \theta_{3}^{n-8} \Delta_{8}+\cdots+c_{k} \theta_{3}^{n-8 k} \Delta_{8}^{k}=1+\left|\Lambda_{1}\right| q+\left|\Lambda_{2}\right| q^{2}+\cdots,
$$

with $c_{k} \neq 0$. (There is no coefficient in front of $\theta_{3}^{n}$, because the series expansion in $q$ begins with $1+\cdots$.) By Proposition [10 the theta series of the shadow is then

$$
\begin{aligned}
\Theta_{\mathcal{S h}(\Lambda)} & =\sum_{m}\left|\mathcal{S h}(\Lambda)_{m}\right| q^{m}=\mathcal{S h} \Theta_{\Lambda} \\
& =\theta_{2}^{n}+c_{1} \theta_{2}^{n-8} \mathcal{S h} \Delta_{8}+\cdots+c_{k} \theta_{2}^{n-8 k} \mathcal{S h} \Delta_{8}^{k} \\
& =d_{1} q^{(n-8 k) / 4}+d_{2} q^{(n-8 k+8) / 4}+\cdots,
\end{aligned}
$$


where $d_{1}=(-1 / 16)^{k} 2^{n-8 k} c_{k} \neq 0$. So, $\mathcal{S h}(\Lambda)_{m} \neq \emptyset$ implies $4 m \equiv n \bmod 8$, and we have $\sigma(\Lambda)=n-8 k$. In particular, if $\Lambda$ is even, we have $\sigma(\Lambda)=0=n-n$, therefore $n$ is a multiple of 8 .

Finally, it follows from the decomposition $\Lambda \simeq \mathbf{Z}^{p} \oplus L$, with $L$ a selfdual lattice of minimum at least 2 , that $\left|\Lambda_{1}\right|=2 p \leqslant 2 n$ with equality if and only if $\Lambda \simeq \mathbf{Z}^{n}$. On the other hand, if $\sigma(\Lambda)=n$ then $\Theta_{\Lambda}=\theta_{3}^{n}=1+2 n q+O\left(q^{2}\right)$, and therefore $\left|\Lambda_{1}\right|=2 n$. Thus $\sigma(\Lambda)=n$ implies $\Lambda \simeq \mathbf{Z}^{n}$.

Remark. Let $\omega>0$ be a multiple of 4 . From Theorem [12] we have $\operatorname{dim} \mathcal{M}_{\omega}^{2,+}=$ $m+1$, where $m=[\omega / 12]$, and there exists a unique $\Theta_{\omega} \in \mathcal{M}_{\omega}^{2,+}$ such that

$$
\Theta_{\omega}(q)=1+a_{2 m+2} q^{2 m+2}+O\left(q^{2 m+4}\right) .
$$

Moreover, it is known that $a_{2 m+2}>0$. Such a theta series is called extremal, and an even selfdual lattice $\Lambda$ of rank $2 \omega$ with $\Theta_{\Lambda}=\Theta_{\omega}$ is called extremal (or, more precisely, extremal of level 1).

The method used in our paper also apply to extremal lattices: see Venk84 and VenMar01 §16]. See also BacVen01, where the case of non-selfdual extremal lattices is also treated.

See [SchSch99] for more informations on extremal lattices.

\section{Computing the theta series of a selfdual lattice}

We can now give more precisely the general form of the theta series of a selfdual lattice.

13. Proposition. Let $\Lambda \subseteq \mathbb{R}^{n}$ be a selfdual lattice with $\sigma(\Lambda)=n-8 k$. Let us write

$$
\Lambda=\mathbf{Z}^{p} \oplus L, \quad L \subseteq \mathbb{R}^{N},
$$

where $L$ is of minimum at least 2 . Then there exist $c_{i} \in \mathbb{Z}$ such that

$$
\Theta_{\Lambda}=\theta_{3}^{n}+c_{1} \Delta_{8} \theta_{3}^{n-8}+c_{2} \Delta_{8}^{2} \theta_{3}^{n-16}+\cdots+c_{k} \Delta_{8}^{k} \theta_{3}^{n-8 k} .
$$

Some values of $c_{i}$ are:

$$
\begin{aligned}
& c_{1}=-2 N \\
& c_{2}=(h-46+2 N) N, \quad \text { where } h:=\left|L_{2}\right| / N \\
& c_{k}=(-1)^{k} 2^{-n+12 k}\left|\mathcal{S h}(\Lambda)_{(n-8 k) / 4}\right| .
\end{aligned}
$$

Proof. As in the proof of Proposition 3 given at the end of the previous Section, we can write

$$
\Theta_{\Lambda}=\theta_{3}^{n}+c_{1} \theta_{3}^{n-8} \Delta_{8}+\cdots+c_{k} \theta_{3}^{n-8 k} \Delta_{8}^{k},
$$

with $c_{i} \in \mathbb{R}$ and $c_{k} \neq 0$, and $k$ is given by $\sigma(\Lambda)=n-8 k$. The first coefficients of the Fourier expansion in $q=e^{i \pi z}$ are

$$
\begin{aligned}
\Theta_{\Lambda}= & \left(1+2 q+2 q^{4}+\cdots\right)^{n}+c_{1}(1+2 q+\cdots)^{n-8}\left(q-8 q^{2}+\cdots\right) \\
& \quad+c_{2}(1+\cdots)^{n-16}(q+\cdots)^{2}+\cdots \\
= & \left(1+2 n q+2 n(n-1) q^{2}+\cdots\right)+c_{1}\left(q+(2 n-24) q^{2}+\cdots\right)+c_{2} q^{2}+\cdots \\
= & 1+\left(2 n+c_{1}\right) q+\left(2 n(n-1)+(2 n-24) c_{1}+c_{2}\right) q^{2}+\cdots
\end{aligned}
$$


In particular, we have $\left|\Lambda_{1}\right|=2 n+c_{1}$. Since $\left|\Lambda_{1}\right|=2 p=2(n-N)$, we have $c_{1}=-2 N$. The second coefficient is then

$$
\begin{aligned}
\left|\Lambda_{2}\right| & =2 n(n-1)+(2 n-24) c_{1}+c_{2} \\
& =2(p+N)(p+N-1)-(2 p+2 N-24) 2 N+c_{2} \\
& =2 p(p-1)+(46-2 N) N+c_{2} .
\end{aligned}
$$

Since $\left|\Lambda_{2}\right|=2 p(p-1)+\left|L_{2}\right|$, this gives $c_{2}=(h-46+2 N) N$ where $h=\left|L_{2}\right| / N$.

By induction on $i$, it is clear that $c_{i}$ is integral.

Now, the theta series of the shadow is

$$
\begin{aligned}
\Theta_{\mathcal{S h}(\Lambda)}=\mathcal{S h} \Theta_{\Lambda} & =c_{k} \theta_{2}^{n-8 k} \mathcal{S h} \Delta_{8}^{k}+\cdots+c_{1} \theta_{2}^{n-8} \mathcal{S h} \Delta_{8}+\theta_{2}^{n} \\
& =c_{k}\left(2 q^{1 / 2}+\cdots\right)^{n-8 k}(-1 / 16+\cdots)^{k}+\cdots \\
& =c_{k} 2^{n-8 k}(-1)^{k} 2^{-4 k} q^{(n-8 k) / 4}+\cdots \\
& =(-1)^{k} 2^{n-12 k} c_{k} q^{(n-8 k) / 4}+\cdots ;
\end{aligned}
$$

this shows that $\left|\mathcal{S h}(\Lambda)_{(n-8 k) / 4}\right|=(-1)^{k} 2^{n-12 k} c_{k}$.

14. Proposition. Let $\Lambda \subseteq \mathbb{R}^{n}$ be a selfdual lattice with $\sigma(\Lambda)=n-8 k$ and of minimum $m$.

(i) For every even positive integer $j$, there exist linear forms $c_{i}: \mathcal{H}^{(2 j)}\left(\mathbb{R}^{n}\right) \rightarrow \mathbb{C}$ such that

$$
\Theta_{\Lambda, P}=\sum_{i=m}^{k+j / 2} c_{i}(P) \Delta_{8}^{i} \theta_{3}^{n+4 j-8 i}, \quad \forall P \in \mathcal{H}^{(2 j)}\left(\mathbb{R}^{n}\right) .
$$

In particular, if $m>k+j / 2$, then $\Theta_{\Lambda, P}=0$ for every $P \in \mathcal{H}^{(2 j)}\left(\mathbb{R}^{n}\right)$.

(ii) For every odd positive integer $j$, there exist linear forms $c_{i}: \mathcal{H}^{(2 j)}\left(\mathbb{R}^{n}\right) \rightarrow \mathbb{C}$ such that

$$
\Theta_{\Lambda, P}=\sum_{i=m}^{k+(j-1) / 2} c_{i}(P) \Phi \Delta_{8}^{i} \theta_{3}^{n+4 j-2-8 i}, \quad \forall P \in \mathcal{H}^{(2 j)}\left(\mathbb{R}^{n}\right) .
$$

In particular, if $m>k+(j-1) / 2$, then $\Theta_{\Lambda, P}=0$ for every $P \in \mathcal{H}^{(2 j)}\left(\mathbb{R}^{n}\right)$.

Proof. We prove only Claim (i), since the proof of Claim (ii) is similar.

Let $j$ be an even positive integer. By Proposition 8 for each $P \in \mathcal{H}^{(2 j)}\left(\mathbb{R}^{n}\right)$, $\Theta_{\Lambda, P}$ is a parabolic form of weight $n / 2+2 j$. By Theorem [12 it is of the form

$$
\Theta_{\Lambda, P}=\sum_{i \geqslant 1} c_{i}(P) \Delta_{8}^{i} \theta_{3}^{n+4 j-8 i} .
$$

Since $\Theta_{\Lambda, P}$ is linear in $P$, the coefficients $c_{i}$ are also linear in $P$.

Let us now suppose that $c_{i} \not \equiv 0$ for some index $i$, and let $a$ [respectively $b$ ] be the smallest [respectively the largest] index $i$ such that $c_{i} \not \equiv 0$. It remains to prove that $a \geqslant m$ and $b \leqslant k+j / 2$. We have

$$
\begin{aligned}
\Theta_{\Lambda, P} & =c_{a}(P) \theta_{3}^{n+4 j-8 a} \Delta_{8}^{a}+\cdots \\
& =c_{a}(P) q^{a}+\cdots
\end{aligned}
$$

hence $c_{a}(P)=\sum_{x \in \Lambda_{a}} P(x)$ is different of zero for some $P \in \mathcal{H}^{(2 j)}\left(\mathbb{R}^{n}\right)$. So, $m \leqslant a$. 
Now, the theta series of the shadow is

$$
\begin{aligned}
\Theta_{\mathcal{S h}(\Lambda), P} & =\mathcal{S h} \Theta_{\Lambda, P}=c_{b}(P) \theta_{2}^{n+4 j-8 b} \mathcal{S} \Delta_{8}^{b}+\cdots \\
& =2^{n+4 j-8 b}(-1 / 16)^{b} c_{b}(P) q^{(n+4 j-8 b) / 4}+\cdots,
\end{aligned}
$$

hence $2^{n+4 j-8 b}(-1 / 16)^{b} c_{b}(P)=\sum_{x \in \mathcal{S h}(\Lambda)_{(n+4 j-8 b) / 4}} P(x)$ is different of zero for some $P \in \mathcal{H}^{(2 j)}\left(\mathbb{R}^{n}\right)$. So, $\sigma(\Lambda)=n-8 k \leqslant n+4 j-8 b$, and therefore $b \leqslant k+$ $j / 2$.

We give now similar statements for even selfdual lattices. (The two propositions above are naturally true also for these lattices; though less precise.) We do not give the proofs, since the arguments are essentially the same as for the equivalent statements for general selfdual lattices.

15. Proposition. Let $\Lambda \subseteq \mathbb{R}^{n}$ be an even selfdual lattice of rank $n=8 N$. Then there exist $c_{i} \in \mathbb{Z}$ such that

$$
\Theta_{\Lambda}=\mathrm{Q}^{N}+c_{1} \Delta_{24} \mathrm{Q}^{N-3}+\cdots+c_{k} \Delta_{24}^{k} \mathrm{Q}^{N-3 k}, \quad k=[N / 3],
$$

with

$$
c_{1}=n(h-30), \quad \text { where } h:=\left|\Lambda_{2}\right| / n .
$$

16. Proposition. Let $\Lambda \subseteq \mathbb{R}^{n}$ be an even selfdual lattice of of rank $n=8 N$ and of minimum $m=2 M$.

(i) For every even positive integer $j$, there exist linear forms $c_{i}: \mathcal{H}^{(2 j)}\left(\mathbb{R}^{n}\right) \rightarrow \mathbb{C}$ such that

$$
\Theta_{\Lambda, P}=\sum_{i=M}^{[(N+j / 2) / 3]} c_{i}(P) \Delta_{24}^{i} \mathrm{Q}^{N+j / 2-3 i}, \quad \forall P \in \mathcal{H}^{(2 j)}\left(\mathbb{R}^{n}\right) .
$$

In particular, if $3 M>N+j / 2$, then $\Theta_{\Lambda, P}=0$ for every $P \in \mathcal{H}^{(2 j)}\left(\mathbb{R}^{n}\right)$.

(ii) For every odd positive integer $j$, there exist linear forms $c_{i}: \mathcal{H}^{(2 j)}\left(\mathbb{R}^{n}\right) \rightarrow \mathbb{C}$ such that

$$
\Theta_{\Lambda, P}=\sum_{i=M}^{[(N+j / 2) / 3]} c_{i}(P) \mathrm{R} \Delta_{24}^{i} \mathrm{Q}^{N+(j-3) / 2-3 i}, \quad \forall P \in \mathcal{H}^{(2 j)}\left(\mathbb{R}^{n}\right) .
$$

In particular, if $3 M>N+(j-3) / 2$, then $\Theta_{\Lambda, P}=0$ for every $P \in \mathcal{H}^{(2 j)}\left(\mathbb{R}^{n}\right)$.

\section{$7 \quad$ Zero coefficients of modular forms}

From Lemma 5 in order to find spherical design strengths of the shells of a lattice, we have to look for vanishing coefficients of $\Theta_{\Lambda, P}$ for $P$ harmonic homogeneous polynomials of different degrees. We give here the results concerning vanishing coefficients of modular forms of the form $\Phi^{\epsilon} \theta_{3}^{\alpha} \Delta_{8}^{\beta}$, since we will meet them several times later. Recall that $\mathbb{N}=\{0,1,2, \ldots\}$.

17. Lemma. Among the coefficients of the modular forms

$$
\Phi^{\epsilon} \theta_{3}^{\alpha} \Delta_{8}^{\beta}=\sum_{m \in \alpha+\mathbb{N}} a_{m} q^{m} \quad \text { and } \quad \mathcal{S h}\left(\Phi^{\epsilon} \theta_{3}^{\alpha} \Delta_{8}^{\beta}\right)=\sum_{m \in \alpha / 4+2 \mathbb{N}} a_{m} q^{m}
$$

where $\epsilon \in\{0,1\}, \alpha \geqslant 0, \beta \geqslant 0$, the following are equal to zero: 

(a) $\left(\theta_{3}^{4} \Delta_{8}\right)^{k}: \quad m-k \equiv 1 \bmod 2, \quad \mathcal{S h}\left(\left(\theta_{3}^{16} \Delta_{8}\right)^{k}\right): m \equiv 2 \bmod 4$;
(b) $\theta_{3}: \quad m \neq a^{2}, \quad \mathcal{S h}\left(\theta_{3}^{2}\right): \quad m \neq\left(a^{2}+b^{2}\right) / 4$
$\mathcal{S h}\left(\theta_{3}\right): \quad \quad \quad \quad \theta_{3}^{3}: a^{2} / 4, \quad m=4^{a}(8 b+7)$,
$\theta_{3}^{2}: \quad m \neq a^{2}+b^{2}, \quad \Phi \theta_{3}^{12}: \quad m=1 ;$
(c) $\theta_{3} \Delta_{8}: \quad m=4^{a}(8 b+5), \quad \Phi \theta_{3}^{3} \Delta_{8}: \quad m=4^{a}(8 b+7)$,
$\theta_{3}^{2} \Delta_{8}: \quad m \neq a^{2}+b^{2}, \quad \Phi \theta_{3}^{16} \Delta_{8}: \quad m=4^{a} 2$,
$\mathcal{S h}\left(\theta_{3}^{2} \Delta_{8}\right): \quad m \neq\left(a^{2}+b^{2}\right) / 4, \quad \mathcal{S h}\left(\Phi \theta_{3}^{16} \Delta_{8}\right): 4^{a} 2$,
$\theta_{3}^{3} \Delta_{8}: \quad \quad m=4^{a}(8 b+7), \quad \operatorname{Sh}\left(\Phi \theta_{3}^{40} \Delta_{8}\right): m=24$,
$\theta_{3}^{7} \Delta_{8}: \quad m=4^{a}(8 b+3) ;$
(d) $\theta_{3}^{5} \Delta_{8}^{2}: \quad m=4^{a}(8 b+1), \quad \Phi \theta_{3}^{20} \Delta_{8}^{2}: \quad m=3$,
$\theta_{3}^{12} \Delta_{8}^{2}: \quad m=4^{a}, \quad \Phi \theta_{3}^{33} \Delta_{8}^{2}: \quad m=4$,
$\Phi \theta_{3}^{8} \Delta_{8}^{2}: \quad \quad \quad \quad \operatorname{Sh}\left(\Phi \theta_{3}^{33} \Delta_{8}^{2}\right): \quad m=49 / 4$,
$\operatorname{Sh}\left(\Phi \theta_{3}^{8} \Delta_{8}^{2}\right): \quad m=4^{a} ;$
(e) $\left.\theta_{3}^{4} \Delta_{8}^{3}: \quad \quad \quad \quad \quad S=4^{a} 2, \quad \Phi \theta_{3}^{24} \Delta_{8}^{3}\right): m=2^{a}$,
$\Phi \theta_{3}^{24} \Delta_{8}^{3}: \quad m=2^{a}$.

Remark. We have checked numerically that, for modular forms of the Lemma with $\beta \leqslant 3$ and $\alpha \leqslant 36$, there is no other zero coefficient $a_{m}$ for $m \leqslant 1200$.

Sketch of the proof. Let $\Theta(z)$ be one of the series of the Lemma.

If $\Theta(z)$ is of the form $\Phi^{\epsilon} \theta_{3}^{\alpha} \Delta_{8}^{\beta}$ or of the form $\mathcal{S h}\left(\Phi^{\epsilon} \theta_{3}^{4 \gamma} \Delta_{8}^{\beta}\right)$, we can express $\Theta(z)$ as a polynomial function of $\theta_{3}(z)$ and $\theta_{4}(z)$. If $\Theta(z)$ is of the form $\mathcal{S h}\left(\Phi^{\epsilon} \theta_{3}^{\alpha} \Delta_{8}^{\beta}\right)$, with $\alpha$ an integer which is not a multiple of 4 , we can express $\Theta(4 z)$ as a polynomial function of $\theta_{3}(z)$ and $\theta_{4}(z)$, by using the formulae of [ConSlo99] Chap. 4, $\S 4.1$, p. 104]. In the sequel of the proof, we suppose that we are in the first case; the second case is treated in a similar way, replacing $\Theta(z)$ by $\Theta(4 z)$.

Let be $\omega:=e^{i \pi / 4}$. For $c$ an integer between 0 and 7 , we define

$$
\begin{aligned}
\Theta_{c}(z) & :=\frac{1}{8} \sum_{k=0}^{7} \omega^{-c k} \Theta\left(\frac{z+k}{4}\right) \\
& =\frac{1}{8} \sum_{k=0}^{7} \omega^{-c k} \sum_{m \geqslant 0} a_{m} e^{i \pi m(z+k) / 4} \\
& =\frac{1}{8} \sum_{k=0}^{7} \omega^{-c k} \sum_{j=0}^{7} \omega^{j k} \sum_{\substack{m \geqslant 0 \\
m \equiv j \bmod 8}} a_{m} e^{i \pi m z / 4} \\
& =\sum_{j=0}^{7} \underbrace{\left(\frac{1}{8} \sum_{k=0}^{7} \omega^{(j-c) k}\right.}_{\substack{0 \text { if } j \neq c \\
1 \text { if } j=c}} \sum_{\substack{m \geqslant 0 \\
m \equiv j \bmod 8}} a^{i \pi m z / 4} \\
& =\sum_{\substack{m \geqslant 0 \\
a_{m} e^{i \pi m z / 4}}} .
\end{aligned}
$$

Therefore, whenever $\Theta_{c}(z)=0$, we have $a_{m}=0$ for $m \equiv c \bmod 8$. Using the identities given in [ConSlo99, Chap. 4, §4.1, p. 104], we can express

$$
\Theta_{c}(z)=\theta_{2}(z)^{d} F\left(\theta_{3}(z), \theta_{4}(z)\right), \quad 0 \leqslant d \leqslant 3, \quad d \equiv c \bmod 8,
$$


where $F$ is a polynomial; so we can check whether $\Theta_{c}(z)=0$. (A computer software like Maple is highly recommended for performing these calculations.)

We have

$$
\begin{gathered}
\Theta_{\text {even }}(z):=\frac{1}{2}(\Theta(z)+\Theta(z+1))=\sum_{m \equiv 0 \bmod 2} a_{m} e^{i \pi m z}, \\
\Theta_{\text {odd }}(z):=\frac{1}{2}(\Theta(z)-\Theta(z+1))=\sum_{m \equiv 1 \bmod 2} a_{m} e^{i \pi m z}, \\
\Theta_{0}(z)=\sum_{m \equiv 0 \bmod 2} a_{4 m} e^{i \pi m z}, \quad \Theta_{4}(z)=\sum_{m \equiv 1 \bmod 2} a_{4 m} e^{i \pi m z},
\end{gathered}
$$

and $\Theta_{\text {even }}, \Theta_{\text {odd }}, \Theta_{0}$ and $\Theta_{4}$ can be expressed in function of $\theta_{3}$ and $\theta_{4}$. If $\Theta_{\text {even }}=0$ [respectively $\Theta_{\text {odd }}=0$ ], then $a_{m}=0$ for $m$ even [resp. odd]. If $\Theta_{\text {even }}$ [respectively $\left.\Theta_{\text {odd }}\right]$ is a multiple of $\Theta_{0}$ [resp. $\left.\Theta_{4}\right]$, we have a relation between $a_{m}$ and $a_{4 m}$ for $m$ even [resp. odd], which allows to decide whenever $a_{4^{k} m}=0$.

These considerations suffice for most of the theta series mentioned in the Lemma. For $\theta_{3}^{2} \Delta_{8}$ [resp. $\mathcal{S h}\left(\theta_{3}^{2} \Delta_{8}\right)$ ], we use Lemma 24 below to show that $a_{m}=0$ whenever the shell of $\mathbf{Z}^{2}$ [resp. $\mathcal{S h}\left(\mathbf{Z}^{2}\right)$ ] of norm $m$ is empty.

The following lemma provides another method to show that certain coefficients of modular forms are nonzero.

18. Lemma. Let $\varphi^{(0)}$ and $\psi$ be two formal series in $q$ with integral coefficients such that

$$
\begin{gathered}
\varphi^{(0)}=a_{k}^{(0)} q^{k}+\sum_{j \geqslant k+1} a_{j}^{(0)} q^{j}, \quad a_{k}^{(0)} \geqslant 1, a_{j}^{(0)} \in \mathbb{Z}, \\
\psi=b_{0}+b_{1} q+\sum_{j \geqslant 2} b_{j} q^{j}, \quad b_{0}, b_{1} \geqslant 1, b_{j} \geqslant 0,
\end{gathered}
$$

and let

$$
\varphi^{(n)}:=\varphi^{(0)} \psi^{n}=\sum_{j \geqslant k} a_{j}^{(n)} q^{j} .
$$

Then the sequence $M_{n}, n \geqslant 0$ defined by

$$
M_{n}:=\max \left\{m \in \mathbb{N} \mid a_{j}^{(n)}>0, k \leqslant j \leqslant m\right\}
$$

is nondecreasing and unbounded.

Proof. We have $M_{0} \geqslant k$. For every $n \geqslant 0$, we have $\varphi^{(n+1)}=\varphi^{(n)} \psi$; thus, for $k \leqslant j \leqslant M_{n}$,

$$
a_{j}^{(n+1)}=a_{j}^{(n)} b_{0}+\sum_{i \geqslant 1} a_{j-i}^{(n)} b_{i} \geqslant a_{j}^{(n)} b_{0}>0 .
$$

Consequently, $M_{n+1} \geqslant M_{n}$. Moreover, for $M=M_{n}$, we have

$$
a_{M+1}^{(n+1)}=a_{M+1}^{(n)} b_{0}+a_{M}^{(n)} b_{1}+\sum_{i \geqslant 2} a_{M+1-i}^{(n)} b_{i} \geqslant a_{M+1}^{(n)} b_{0}+a_{M}^{(n)} b_{1}>a_{M+1}^{(n)} .
$$

Consequently, $a_{M+1}^{(n+1)} \geqslant a_{M+1}^{(n)}+1$, since all coefficients are integers. Similarly,

$$
a_{M+1}^{(n+h)} \geqslant a_{M+1}^{(n+h-1)}+1 \geqslant \cdots \geqslant a_{M+1}^{(n)}+h .
$$

Therefore, for $h$ large enough, we have $a_{M+1}^{(n+h)}>0$, and then $M_{n+h} \geqslant M_{n}+1$. That shows that the sequence $M_{n}$ is unbounded. 


\section{Root systems of norm 2}

For analysing selfdual lattices of rank at most 24 , we need some informations on the first shells of a selfdual lattice $\Lambda$. The decomposition $\Lambda=\mathbf{Z}^{p} \oplus L$ gives us the exact form of the shell $\Lambda_{1}$; we give now the form of $\Lambda_{2}$.

Let us first recall that a root system is a subset $R \subseteq \mathbb{R}^{n}$ such that

(i) $R$ is finite and does not contain 0 ;

(ii) for every $x, y \in R$, we have $\mathrm{r}_{y}(x) \in R$, where $\mathrm{r}_{y}$ is the reflection of axis $\mathbb{R} y$;

(iii) for every $x, y \in R$, the number $2\langle x \mid y\rangle /\langle x \mid x\rangle$ is an integer.

Warning. In the usual definition, it is required that $R$ span $\mathbb{R}^{n}$ as a vector space. Here, we leave out this condition in order to simplify the formulation of our results; in particular, we consider the empty set in $\mathbb{R}^{n}$ as a root system.

19. Lemma - Definition. Let $\Lambda$ be an integral lattice. Then $\Lambda_{2}$ is a root system, called the root system of $\Lambda$.

Proof. For $x$ and $y \in \Lambda_{2}$, we have $\mathrm{r}_{y}(x)=x-\langle x \mid y\rangle y$. Therefore, since $\langle x \mid y\rangle$ is an integer, we have $\mathrm{r}_{y}(x) \in \Lambda_{2}$. Moreover, $2\langle x \mid y\rangle /\langle x \mid x\rangle=\langle x \mid y\rangle$ is clearly an integer.

If $\Lambda$ is a selfdual lattice of minimum at least 2 and of root system $R$, we write sometimes $\Lambda=R^{+}$. (It happens that this notation is unambiguous up to rank 23, that is there is at most one selfdual lattice of minimum at least 2 whose root system is a given root system in $\mathbb{R}^{n}, n \leqslant 23$.)

Let us now recall some classical facts on root systems. If $R \subseteq \mathbb{R}^{p}$ and $S \subseteq \mathbb{R}^{q}$ are root systems, their orthogonal union is the root system in $\mathbb{R}^{p+q}$ defined by

$$
R+S:=(R \oplus\{0\}) \cup(\{0\} \oplus S) \subseteq \mathbb{R}^{p} \oplus \mathbb{R}^{q} .
$$

We write $k R$ for $R+\cdots+R, k$ terms. A root system is called irreducible if it is not an orthogonal union of smaller root systems. Clearly, any root system can be written uniquely (up to permutation of the terms) as an orthogonal union of irreducible root systems. Note that a nonempty irreducible root system always spans its ambient space. The only empty irreducible root system is of dimension 1, and is noted $\mathbf{O}_{1}$.

An important number for an irreducible root system $R$ is its Coxeter number, which is the integer $h$ satisfying the relation

$$
|R|=n h .
$$

If $R$ is empty, we have $h=0$.

The list of irreducible root systems is well known. We give here the list of those of norm 2, that is those root systems $R$ that verify $\langle x \mid x\rangle=2$ for every $x \in R$ (they are also called simply laced root systems by some authors). In the notation, the index indicates the dimension of the space where the root system lies.

$$
\begin{array}{llll}
\mathbf{O}_{1}, & h=0 ; & \mathbf{E}_{6}, & h=12 ; \\
\mathbf{A}_{n}, n \geqslant 1, & h=n+1 ; & \mathbf{E}_{7}, & h=18 ; \\
\mathbf{D}_{n}, n \geqslant 4, \quad h=2(n-1) ; & \mathbf{E}_{8}, & h=30 .
\end{array}
$$

We denote by $\mathbf{O}_{n}:=n \mathbf{O}_{1}$ the empty root system in $\mathbb{R}^{n}$.

The following definition, justified by the next lemma, is inspired by the corresponding notion for lattices (see MartV01 pp. 28ff]). 
20. Definition. A root system $R$ is called strongly eutactic if, in its decomposition in irreducible root systems, all irreducible components have the same Coxeter number. In this case, we define the Coxeter number of $R$ as the Coxeter number of any of its irreducible components.

A nonempty strongly eutactic root system spans its ambiant space. Note that the equality $|R|=n h$ holds for strongly eutactic root systems.

21. Lemma. Let $R$ be a root system of norm 2 that is a spherical 3-design. Then $R$ is strongly eutactic.

The converse is true also: see Proposition 23$] \mathrm{i}$.

Proof. Let us write a root system $R \subseteq \mathbb{R}^{n}$ as

$$
R=R_{1}+R_{2}+\cdots+R_{k},
$$

where the $R_{i}$ 's are irreducible. Let $V_{i}$ be the subspace of $\mathbb{R}^{n}$ where $R_{i}$ lies (thus $\left.\mathbb{R}^{n}=V_{1} \oplus V_{2} \oplus \cdots \oplus V_{k}\right)$, and let $n_{i}=\operatorname{dim} V_{i}$. Any point $x \in \mathbb{R}^{n}$, is written uniquely as

$$
x=x_{1}+x_{2}+\cdots+x_{k}, \quad x_{i} \in V_{i} .
$$

Let us consider the harmonic polynomials of degree 2 defined by

$$
f_{i, j}(x)=\frac{1}{2 n_{i}}\left\langle x_{i} \mid x_{i}\right\rangle-\frac{1}{2 n_{j}}\left\langle x_{j} \mid x_{j}\right\rangle .
$$

We have

$$
\sum_{x \in R} f_{i, j}(x)=\frac{2\left|R_{i}\right|}{2 n_{i}}-\frac{2\left|R_{j}\right|}{2 n_{j}}=h_{i}-h_{j} .
$$

So, if $R$ is a spherical 3-design, we must have $\sum_{x \in R} f_{i, j}(x)=0$ and therefore $h_{i}=h_{j}$; in other words, $R$ is strongly eutactic.

We recall now the notion of reproducing kernel, which will help us to analyse strongly eutactic root systems:

Let $\mathcal{H}$ be a complex (or a real) finite-dimensional Hilbert space of functions on a nonempty set $\Omega$. We use the convention that hermitian scalar products are antilinear in the first variable. There exists a unique function $\Phi: \Omega \times \Omega \rightarrow \mathbb{C}$, called reproducing kernel, such that $\Phi(x, \cdot) \in \mathcal{H}$ for all $x \in \Omega$, and

$$
f(x)=\langle\Phi(x, \cdot) \mid f\rangle, \quad \forall f \in \mathcal{H}, \forall x \in \Omega .
$$

This kernel verifies $\overline{\Phi(y, x)}=\Phi(x, y)$. It is of positive type; that is, for any finitely supported function $\Omega \rightarrow \mathbb{C}, y \mapsto \lambda_{y}$,

$$
\sum_{x, y \in \Omega} \overline{\lambda_{x}} \lambda_{y} \Phi(x, y) \geqslant 0
$$

Moreover, the set $\{\Phi(x, \cdot) \mid x \in \Omega\}$ generates $\mathcal{H}$. For all this, see for example BekHar02.

The positivity of $\Phi$ implies the following result:

22. Lemma. Let $\Omega \rightarrow \mathbb{C}, y \mapsto \lambda_{y}$ be a finitely supported function. Then

$$
\sum_{x, y \in \Omega} \overline{\lambda_{x}} \lambda_{y} \Phi(x, y)=0 \quad \text { if and only if } \quad \sum_{y \in \Omega} \lambda_{y} f(y)=0, \quad \forall f \in \mathcal{H} .
$$


Proof. Let $\Omega^{\prime}$ be the vector space of finitely supported functions on $\Omega$. We define a hermitian form $h$ on $\Omega^{\prime}$ by:

$$
h(\mu, \lambda)=\sum_{x, y \in \Omega} \overline{\mu_{x}} \lambda_{y} \Phi(x, y), \quad \mu, \lambda \in \Omega^{\prime} .
$$

For $x \in \Omega$, let $\delta_{x} \in \Omega^{\prime}$ denote the function on $\Omega$ that takes value 1 at $x$ and value 0 elsewhere. Let $\Omega_{0}^{\prime}$ be the set of $\lambda \in \Omega^{\prime}$ such that $\langle\mu \mid \lambda\rangle=0$ for every $\mu \in \Omega^{\prime}$. Then $\lambda \in \Omega_{0}^{\prime}$ if and only if $h\left(\delta_{x}, \lambda\right)=0$ for all $x \in X$, if and only if

$$
\sum_{y \in \Omega} \lambda_{y} \Phi(x, y)=0, \quad \forall x \in \Omega .
$$

But since $\{\Phi(x, \cdot) \mid x \in \Omega\}$ generates $\mathcal{H}$, the last condition is equivalent to

$$
\sum_{y \in \Omega} \lambda_{y} f(y)=0, \quad \forall f \in \mathcal{H} .
$$

Now, the positivity of $\Phi$ implies that

$$
h(\lambda, \lambda)=0 \quad \text { if and only if } \quad \lambda \in \Omega_{0}^{\prime} .
$$

This is exactly what is claimed by the Lemma.

Let us now consider the following special case:

$$
\begin{gathered}
\Omega=\mathbb{S}^{n-1}, \quad \mathcal{H}=\mathcal{H}^{(j)}\left(\mathbb{R}^{n}\right), \\
\langle f \mid g\rangle=\int_{\mathbb{S}^{n-1}} \overline{f(u)} g(u) d \sigma(u), \quad f, g \in \mathcal{H}^{(j)}\left(\mathbb{R}^{n}\right),
\end{gathered}
$$

where $\sigma$ is the probability measure on $\mathbb{S}^{n-1}$ invariant by rotation. Let $\Phi^{(j)}$ be the corresponding reproducing kernel. It is known that

$$
\Phi^{(j)}(x, y)=\mathrm{Q}^{(j)}(\langle x \mid y\rangle) \quad \forall x, y \in \mathbb{S}^{n-1},
$$

where $\mathrm{Q}^{(j)}(t)$ is an appropriate Gegenbauer polynomials, with the normalisation $\mathrm{Q}^{(j)}(1)=\operatorname{dim}\left(\mathcal{H}^{(j)}\left(\mathbb{R}^{n}\right)\right)$; see [DeGoSe77] and Vile68, §IX.3]. We have, for example,

$$
\begin{gathered}
\mathrm{Q}^{(0)}(t)=1, \quad \mathrm{Q}^{(1)}(t)=n t, \quad \mathrm{Q}^{(2)}(t)=\frac{n+2}{2}\left(n t^{2}-1\right), \\
\mathrm{Q}^{(3)}(t)=\frac{n(n+4)}{6}\left((n+2) t^{3}-3 t\right), \\
\mathrm{Q}^{(4)}(t)=\frac{n(n+6)}{24}\left((n+2)(n+4) t^{4}-6(n+2) t+3\right) .
\end{gathered}
$$

We can now prove:

23. Proposition. Let $R$ be a nonempty strongly eutactic root system of norm 2 . Let us consider the conditions

$$
\sum_{x \in R} f(x)=0, \quad \forall f \in \mathcal{H}^{(2 j)}\left(\mathbb{R}^{n}\right) .
$$

Then:

(i) Condition $\left(C_{2}\right)$ always holds (equivalently, nonempty strongly eutactic root systems are spherical 3-designs); 
(ii) Condition $\left(C_{4}\right)$ holds if and only if $R$ is equivalent to one of the following systems:

$$
\mathbf{A}_{1}, \quad \mathbf{A}_{2}, \quad \mathbf{D}_{4}, \quad \mathbf{E}_{6}, \quad \mathbf{E}_{7}, \quad \mathbf{E}_{8}
$$

(iii) Condition $\left(C_{6}\right)$ holds if and only if $R$ is equivalent to one of the following systems:

$$
\mathbf{A}_{1}, \quad 2 \mathbf{A}_{1}, \quad \mathbf{E}_{8}, \quad 2 \mathbf{E}_{8}, \quad \mathbf{D}_{16}
$$

(iv) Condition $\left(C_{8}\right)$ holds if and only if $R$ is equivalent to one of the following systems:

$$
\mathbf{A}_{1}, \quad \mathbf{A}_{2}
$$

(v) Condition $\left(C_{10}\right)$ holds if and only if $R$ is equivalent to one of the following systems:

$$
\mathbf{A}_{1}, \quad 2 \mathbf{A}_{1}, \quad \mathbf{A}_{2} \quad \mathbf{D}_{4}, \quad \mathbf{E}_{8}
$$

(vi) Condition $\left(C_{12}\right)$ holds if and only if $R$ is equivalent to the system

\section{$\mathbf{A}_{1}$.}

Proof. For $x$ and $y \in R$, we have $\langle x \mid y\rangle \in\{0, \pm 1, \pm 2\}$. Let $x \in R$, and let, for $\alpha \in\{0, \pm 1, \pm 2\}$,

$$
N_{\alpha}:=|\{y \in \mathrm{R} \mid\langle x \mid y\rangle=\alpha\}| .
$$

We have the evident relations $N_{-\alpha}=N_{\alpha}, N_{2}=1$, and $N_{0}+2 N_{1}+2 N_{2}=n h$. Moreover, it is known that $N_{\alpha}$ is independent of $x$ and that

$$
N_{1}=2 h-4
$$

(see Bourbaki Bour81, Lie VI, $\S 1.11$, prop. 3.2, where it is stated for (nonempty) irreducible root systems; but it immediately extends to nonempty strongly eutactic root systems).

Now, we renormalize $R$ by $\widetilde{R}=\frac{1}{\sqrt{2}} R$, so that $\widetilde{R} \subseteq \mathbb{S}^{n-1}$. By Lemma 22 (applied with $\lambda_{x}=1$ if $x \in X$ and $\lambda_{x}=0$ otherwise) and equation (*), Condition $\left(C_{2 j}\right)$ is equivalent to

$$
\sum_{x, y \in \widetilde{R}} \mathrm{Q}^{(2 j)}(\langle x \mid y\rangle)=0 .
$$

However, since for every $x \in R$ we have [note that $\mathrm{Q}^{(2 j)}(-\alpha)=\mathrm{Q}^{(2 j)}(\alpha)$ ]:

$$
\begin{aligned}
\sum_{y \in \widetilde{R}} \mathrm{Q}^{(2 j)}(\langle x \mid y\rangle) & =\sum_{\alpha} N_{\alpha} \mathrm{Q}^{(2 j)}(\alpha / 2) \\
& =2 \mathrm{Q}^{(2 j)}(1)+(4 h-8) \mathrm{Q}^{(2 j)}(1 / 2)+(n h-4 h+6) \mathrm{Q}^{(2 j)}(0),
\end{aligned}
$$

Condition $\left(C_{2 j}\right)$ is equivalent to

$$
2 \mathrm{Q}^{(2 j)}(1)+(4 h-8) \mathrm{Q}^{(2 j)}(1 / 2)+(n h-4 h+6) \mathrm{Q}^{(2 j)}(0)=0 .
$$

This condition is linear in $h$ and polynomial in $n$ ( since $\mathrm{Q}^{(2 j)}$ is polynomial in $n$ ). It is, for $2 j \leqslant 12$ :

$$
\begin{gathered}
0=0 \\
n(n+4)(n+6)((n-10) h+6(n+2))=0 \\
n(n+2)(n+6)(n+10)\left(\left(n^{2}-48 n+272\right) h+30(n-4)(n+4)\right)=0
\end{gathered}
$$




$$
\begin{aligned}
& n(n+2)(n+4)(n+8)(n+14) \\
& \quad \times\left((n-4)(n-30)(n-50) h+42(n+6)\left(3 n^{2}-14 n+40\right)\right)=0
\end{aligned}
$$

$\left(C_{10}\right) \quad(n-2) n(n+2)(n+4)(n+6)(n+10)(n+18)$

$$
\times\left((n-24)(n-28)(n-76) h+30(n+8)\left(17 n^{2}-8 n+336\right)\right)=0
$$

$$
\begin{aligned}
& n(n+2)(n+4)(n+6)(n+8)(n+12)(n+22) \\
& \times\left(\left(n^{5}-186 n^{4}+10852 n^{3}-228504 n^{2}+1659232 n-967680\right) h\right. \\
& \left.+66 n(n-2)(n+10)\left(31 n^{2}+130 n+1144\right)\right)=0
\end{aligned}
$$

We can now explicit the positive integral solutions of these equations. Let us take for example $\left(C_{4}\right)$ : we have $(n-10) h+6(n+2)=0$, hence

$$
h=\frac{6(n+2)}{10-n} \text {. }
$$

Since $h>0$, we have $n<10$. Now, if we introduce $n=1,2, \ldots, 9$ in the last formula, we obtain the integral solutions

$$
(n, h)=(1,2),(2,3),(4,6),(6,12),(7,18),(8,30),(9,16) .
$$

It is now easy to list all strongly eutactic root systems having one of these parameters.

\section{B Application to some selfdual lattices}

\section{The cubic lattices}

Let

$$
\mathbf{Z}^{n}:=\left\{x=\left(x_{1}, \ldots, x_{n}\right) \in \mathbb{R}^{n} \mid x_{i} \in \mathbb{Z}, i=1, \cdots, n\right\}
$$

be the cubic lattice of rank $n$. It is an odd selfdual lattice whose shadow is:

$$
\mathcal{S h}\left(\mathbf{Z}^{n}\right)=\left\{x \in \mathbb{R}^{n} \mid x_{i} \in \mathbb{Z}+\frac{1}{2}, i=1, \cdots, n\right\} .
$$

In particular, $\sigma\left(\mathbf{Z}^{n}\right)=n$.

24. Lemma. We have

$$
\Theta_{\mathbf{Z}^{n}, P}= \begin{cases}\theta_{3}^{n} & \text { if } P=1, \\ 0 & \text { if } P \in \mathcal{H}^{(2)}\left(\mathbb{R}^{n}\right), \\ c_{1}(P) \Delta_{8} \theta_{3}^{n} & \text { if } P \in \mathcal{H}^{(4)}\left(\mathbb{R}^{n}\right), \\ c_{2}(P) \Phi \Delta_{8} \theta_{3}^{n} & \text { if } P \in \mathcal{H}^{(6)}\left(\mathbb{R}^{n}\right),\end{cases}
$$

where $c_{1}$ is a nonzero linear form if and only if $n \geqslant 2$, and $c_{2}$ is a nonzero linear form if and only if $n \geqslant 3$.

Remark. The theta series of $\mathcal{S h}\left(\mathbf{Z}^{n}\right)$ are given by Proposition 10,

Proof. The equality $\Theta_{\mathbf{Z}^{n}}=\theta_{3}^{n}$ follows directly from $\Theta_{\mathbf{Z}^{n}}=\left(\Theta_{\mathbf{Z}}\right)^{n}$ and $\Theta_{\mathbf{Z}}=\theta_{3}$. Proposition 14 gives the form of $\Theta_{\mathbf{Z}^{n}, P}$ for $P \in \mathcal{H}^{(2 j)}\left(\mathbb{R}^{n}\right), j=1,2,3$.

It remains to show that $c_{1}$ and $c_{2}$ are not identically zero for $n$ large enough, i.e., there exists $P \in \mathcal{H}^{(4)}\left(\mathbb{R}^{n}\right)$ (if $n \geqslant 2$ ) such that $\Theta_{\mathbf{Z}^{n}, P} \neq 0$, and there exists $Q \in \mathcal{H}^{(6)}\left(\mathbb{R}^{n}\right)$ (if $n \geqslant 3$ ) such that $\Theta_{\mathbf{Z}^{n}, Q} \neq 0$. We can chose for example

$$
P(x)=x_{1}^{4}+x_{2}^{4}-6 x_{1}^{2} x_{2}^{2}, \quad \Theta_{\mathbf{Z}^{n}, P}(z)=4 q+\cdots,
$$


and

$$
\begin{gathered}
Q(x)=\left(x_{1}^{6}+x_{2}^{6}+x_{3}^{6}\right)-15\left(x_{1}^{4} x_{2}^{2}+x_{2}^{4} x_{3}^{2}+x_{3}^{4} x_{1}^{2}\right)+90\left(x_{1}^{2} x_{2}^{2} x_{3}^{2}\right), \\
\Theta_{\mathbf{Z}^{n}, Q}(z)=6 q+\cdots
\end{gathered}
$$

Finally, the fact that $c_{1} \equiv 0$ [respectively $c_{2} \equiv 0$ ] if $n=1$ [respectively if $n=1$ or 2$]$ is an easy exercise.

The zero coefficients of the Fourier expansion of the series of the last Lemma are given in Lemma 17 Therefore, by Lemma 5 we have:

\section{THEOREM.}

(i) For $n \geqslant 2$, all nonempty shells of $\mathbf{Z}^{n}$ and $\mathcal{S h}\left(\mathbf{Z}^{n}\right)$ are spherical 3-designs.

(ii) The following shells are spherical $3^{1} / 2^{\text {-designs: }}$

$$
\begin{array}{ll}
\mathbf{Z}^{2} \text { and } \mathcal{S h}\left(\mathbf{Z}^{2}\right): & \text { every nonempty shell, } \\
\left(\mathbf{Z}^{16}\right)_{m}: & m=4^{a} 2, a \geqslant 0 \\
\mathcal{S h}\left(\mathbf{Z}^{16}\right)_{m}: & m=4^{a} 2, a \geqslant 1 \\
\mathcal{S h}\left(\mathbf{Z}^{40}\right)_{m}: & m=24 .
\end{array}
$$

(iii) The following shells are spherical 5-designs:

$$
\begin{array}{ll}
\left(\mathbf{Z}^{4}\right)_{m}: & m=2 a, a \geqslant 1 \\
\left(\mathbf{Z}^{7}\right)_{m}: & m=4^{a}(8 b+3), a, b \geqslant 0 \\
\mathcal{S h}\left(\mathbf{Z}^{16}\right)_{m}: & m=4 a+2, a \geqslant 1 .
\end{array}
$$

Note that the zero coefficients of series $\Delta_{8} \theta_{3}^{n}$ for $n=2,3$, and $\Phi_{8} \theta_{3}^{n}$ for $n=3$, that are mentioned in Lemma 17 correspond to empty shells of the lattices $\mathbf{Z}^{2}$ and $\mathbf{Z}^{3}$. Zero coefficients of $\Delta_{8} \theta_{3}^{n}$ for $n=1$ and $\Phi \Delta_{8} \theta_{3}^{n}$ for $n=1,2$ are irrelevant for our purpose, because they correspond to cases where $c_{1}$ and $c_{2}$ are zero in Lemma 24. Similar remarks hold for the shadows of these modular forms.

We give in Appendix an alternative proof that some shells of the cubic lattices of rank 4 and 7 are 5 -designs.

\section{Proposition.}

(i) For $n \geqslant 2$ and $1 \leqslant m \leqslant 1200$, the nonempty shells of norm $m$ of $\mathbf{Z}^{n}$ are not spherical 5-designs, except those mentioned in Theorem 25

(ii) For $n \geqslant 2$ and $n / 4 \leqslant m \leqslant n / 4+1200$, the nonempty shells of norm $m$ of $\mathcal{S h}\left(\mathbf{Z}^{n}\right)$ are not a spherical 5-designs, except those mentioned in Theorem 25

Proof. (i) We check first numerically that the statement holds for $n<408$. Then, we apply Lemma 18 with $\varphi^{(0)}=\Delta_{8}(z)=q+O\left(q^{2}\right)$ and $\psi=\theta_{3}(z)=1+2 q+O\left(q^{4}\right)$. Numerical computations give $M_{408} \geqslant 1200$, and thus the coefficients $a_{j}^{(n)}$ of

$$
\varphi^{(n)}=\Delta_{8}(z) \theta_{3}(z)^{n}=\sum_{j \geqslant 1} a_{j}^{(n)} q^{j}
$$

are positive for $1 \leqslant j \leqslant 1200$ and $n \geqslant 408$. By Lemma 24 and Lemma 5 the corresponding shells $\left(\mathbf{Z}^{n}\right)_{j}$ are not spherical 5-designs. 
(ii) We check first numerically that the statement holds for $n<426$. Then, we apply Lemma 18 to

$$
\begin{gathered}
\varphi^{(0)}=-16 \mathcal{S h} \Delta_{8}(z / 2)=1-16 q+O\left(q^{2}\right), \\
\psi=q^{-1 / 8} \theta_{2}(z / 2)=2+2 q+O\left(q^{3}\right) .
\end{gathered}
$$

(The substitution $z \mapsto z / 2$ is equivalent to the substitution $q \mapsto q^{1 / 2}$.) Numerical computations give $M_{426} \geqslant 600$. It follows that the coefficients $a_{j}^{(n)}$ of

$$
\varphi^{(n)}=-16 q^{n / 8} \operatorname{Sh} \Delta_{8}(z / 2) \theta_{2}(z / 2)^{n}=\sum_{j \geqslant 0} a_{j}^{(n)} q^{j}
$$

are positive for $0 \leqslant j \leqslant 600$ and $n \geqslant 426$. In other words, the coefficients $c_{j}^{(n)}$ of

$$
\operatorname{Sh} \Delta_{8}(z) \theta_{2}(z)^{n}=\sum_{j \geqslant 0} c_{j}^{(n)} q^{2 j+n / 4}
$$

are negative for $n / 4 \leqslant 2 j+n / 4 \leqslant n / 4+1200$ and $n \geqslant 426$. The proof is achieved by invoking Lemma 24 together with Proposition [10 and Lemma 5

\section{The Witt lattices}

Let $n$ be a positive multiple of 4 . The Witt lattice of rank $n$ is the lattice

$$
\Gamma_{n}:=\left\{\begin{array}{l|l}
x=\left(x_{1}, \ldots, x_{n}\right) \in \mathbb{R}^{n} & \begin{array}{l}
2 x_{i} \in \mathbb{Z} \quad \forall i, \\
x_{i}-x_{1} \in \mathbb{Z} \quad \forall i, \\
x_{1}+x_{2}+\cdots+x_{n} \in 2 \mathbb{Z}
\end{array}
\end{array}\right\} .
$$

It is selfdual, and it is even if $n \equiv 0 \bmod 8$ and odd otherwise. When $n \equiv 4 \bmod 8$, the shadow of $\Gamma_{n}$ is

$$
\mathcal{S h}\left(\Gamma_{n}\right)=\left\{\begin{array}{l|l}
x=\left(x_{1}, \ldots, x_{n}\right) \in \mathbb{R}^{n} & \begin{array}{l}
2 x_{i} \in \mathbb{Z} \quad \forall i \\
x_{i}-x_{1} \in \mathbb{Z} \quad \forall i, \\
x_{1}+x_{2}+\cdots+x_{n} \in 2 \mathbb{Z}+1
\end{array}
\end{array}\right\}
$$

The lattice $\Gamma_{4}$ is equivalent to the cubic lattice $\mathbf{Z}^{4}$ and is thus analysed in the previous Section; therefore, we assume that $n \geqslant 8$. The lattice $\Gamma_{8}$ is analysed more precisely in the next Section.

27. Lemma. Let $n$ be a multiple of 4 greater than or equal to 8 . Then

$$
\Theta_{\Lambda, P}= \begin{cases}\frac{1}{2}\left(\theta_{2}^{n}+\theta_{3}^{n}+\theta_{4}^{n}\right) & \text { if } P=1, \\ 0 & \text { if } P \in \mathcal{H}^{(2)}\left(\mathbb{R}^{n}\right), \\ c_{1}(P) \theta_{2}^{4} \theta_{3}^{4} \theta_{4}^{4}\left(-\theta_{2}^{n-4}+\theta_{3}^{n-4}-\theta_{4}^{n-4}\right) & \text { if } P \in \mathcal{H}^{(4)}\left(\mathbb{R}^{n}\right), \\ c_{2}(P) \theta_{2}^{4} \theta_{3}^{4} \theta_{4}^{4}\left(\left(\theta_{3}^{4}+\theta_{4}^{4}\right) \theta_{2}^{n-4}\right. & \\ \left.\quad+\left(\theta_{4}^{4}-\theta_{2}^{4}\right) \theta_{3}^{n-4}-\left(\theta_{2}^{4}+\theta_{3}^{4}\right) \theta_{4}^{n-4}\right) & \text { if } P \in \mathcal{H}^{(6)}\left(\mathbb{R}^{n}\right) .\end{cases}
$$

where $c_{1}$ and $c_{2}$ are nonzero linear forms on $\mathcal{H}^{(4)}\left(\mathbb{R}^{n}\right)$ and $\mathcal{H}^{(6)}\left(\mathbb{R}^{n}\right)$ respectively.

Proof. Let $G$ the subgroup of $\mathrm{O}(n)$ containing the transformations of the form

$$
\left(x_{1}, \ldots, x_{n}\right) \mapsto\left(\epsilon_{1} x_{1}, \ldots, \epsilon_{n} x_{n}\right)
$$


where $\epsilon_{i}= \pm 1$ and $\epsilon_{1} \epsilon_{2} \cdots \epsilon_{n}=1$. It is clear that $G$ leaves $\Gamma_{n}$ invariant. We denote by $\mathcal{H}^{(2 j)}\left(\mathbb{R}^{n}\right)^{G}$ the set of elements of $\mathcal{H}^{(2 j)}\left(\mathbb{R}^{n}\right)$ which are invariant under the action of $G$ given by $(\gamma P)(x)=P\left(\gamma^{-1} x\right)$. Let

$$
\pi: \mathcal{H}^{(2 j)}\left(\mathbb{R}^{n}\right) \rightarrow \mathcal{H}^{(2 j)}\left(\mathbb{R}^{n}\right)^{G}, \quad \pi(P)=\frac{1}{|G|} \sum_{\gamma \in G} \gamma P
$$

be the projection on the invariant part of $\mathcal{H}^{(2 j)}\left(\mathbb{R}^{n}\right)$. Since for all $\gamma \in G$

$$
\Theta_{\Gamma_{n}, \gamma P}=\Theta_{\gamma^{-1} \Gamma_{n}, P}=\Theta_{\Gamma_{n}, P}
$$

we have $\Theta_{\Gamma_{n}, P}=\Theta_{\Gamma_{n}, \pi(P)}$. Therefore, it suffices to prove the Lemma for $P \in$ $\mathcal{H}^{(2 j)}\left(\mathbb{R}^{n}\right)^{G}$.

Let

$$
\Gamma_{n}^{\prime}=\left\{\begin{array}{l|l}
x=\left(x_{1}, \ldots, x_{n}\right) \in \mathbb{R}^{n} & \begin{array}{l}
2 x_{i} \in \mathbb{Z} \quad \forall i, \\
x_{i}-x_{1} \in \mathbb{Z} \quad \forall i, \\
-x_{1}+x_{2}+\cdots+x_{n} \in 2 \mathbb{Z}
\end{array}
\end{array}\right\},
$$

which is a lattice equivalent to $\Gamma_{n}$. Let $\mathbf{D}_{n}:=\left\{x \in \mathbf{Z}^{n} \mid\langle x \mid x\rangle \in 2 \mathbb{Z}\right\}$; we have, by definition, $\operatorname{Sh}\left(\mathbf{Z}^{n}\right)=\mathbf{D}_{n}^{\sharp} \backslash \mathbf{D}_{n}$. We have the following inclusions of lattices, where labels indicate indices of sublattices:



Expressing $\mathbf{D}_{n}^{\sharp}$ as the union of its classes modulo $\mathbf{D}_{n}$, we obtain

$$
\mathbf{D}_{n}^{\sharp}=\left(\Gamma_{n} \backslash \mathbf{D}_{n}\right) \sqcup\left(\mathbf{Z}^{n} \backslash \mathbf{D}_{n}\right) \sqcup\left(\Gamma_{n}^{\prime} \backslash \mathbf{D}_{n}\right) \sqcup \mathbf{D}_{n} .
$$

Hence, since $\mathcal{S h}\left(\mathbf{Z}^{n}\right)=\mathbf{D}_{n}^{\sharp} \backslash \mathbf{Z}^{n}$,

$$
\mathcal{S h}\left(\mathbf{Z}^{n}\right)=\left(\Gamma_{n} \backslash \mathbf{D}_{n}\right) \sqcup\left(\Gamma_{n}^{\prime} \backslash \mathbf{D}_{n}\right),
$$

from which we deduce,

$$
\Theta_{\mathcal{S h}\left(\mathbf{Z}^{n}\right), P}=\Theta_{\Gamma_{n}, P}+\Theta_{\Gamma_{n}^{\prime}, P}-2 \Theta_{\mathbf{D}_{n}, P} .
$$

As $2 \Theta_{\mathbf{D}_{n}, P}(z)=\Theta_{\mathbf{Z}^{n}, P}(z)+\Theta_{\mathbf{Z}^{n}, P}(z+1)$, we have

$$
\Theta_{\Gamma_{n}, P}(z)+\Theta_{\Gamma_{n}^{\prime}, P}(z)=\Theta_{\mathbf{Z}^{n}, P}(z)+\Theta_{\mathbf{Z}^{n}, P}(z+1)+\Theta_{\mathcal{S h}\left(\mathbf{Z}^{n}\right), P}(z) .
$$

Let $\sigma$ be the orthogonal transformation of $\mathbb{R}^{n}$ defined by

$$
\sigma\left(x_{1}, x_{2}, \ldots, x_{n}\right)=\left(-x_{1}, x_{2}, \ldots, x_{n}\right) ;
$$

we have $\sigma \Gamma_{n}^{\prime}=\Gamma_{n}$. Let $P \in \mathcal{H}^{(2 j)}\left(\mathbb{R}^{n}\right)^{G}$, where $0 \leqslant 2 j \leqslant 6$. It is easily checked that, since $2 j \leqslant n, P$ is a polynomial which is even in $x_{1}, x_{2}, \ldots, x_{n}$, and therefore $P$ is invariant under the action of $\sigma$. Thus, $\Theta_{\Gamma_{n}^{\prime}, P}=\Theta_{\Gamma_{n}, \sigma P}=\Theta_{\Gamma_{n}, P}$, and

$$
\Theta_{\Gamma_{n}, P}(z)=\frac{1}{2}\left(\Theta_{\mathbf{Z}^{n}, P}(z)+\Theta_{\mathbf{Z}^{n}, P}(z+1)+\Theta_{\mathcal{S h}\left(\mathbf{Z}^{n}\right), P}(z)\right) .
$$

We use Lemma 24 and identities found in ConSlo99 Chap. 4, §4.1, p. 104] to conclude. 
Now, using Lemma [5] we have:

28. Theorem. Let $n$ be a multiple of 4 greater than or equal to 8.

(i) All nonempty shells of $\Gamma_{n}$ and $\mathcal{S h}\left(\Gamma_{n}\right)$ are spherical 3-designs.

(ii) All nonempty shells of $\Gamma_{8}$ are spherical 7-designs.

(iii) All nonempty shells of $\Gamma_{16}$ are spherical $3^{1 / 2}$-designs.

We do not know if any shell of $\Gamma_{n}$, for $n \geqslant 12$ is a spherical 5-design; however, as in the case of cubic lattices, we can show:

29. Proposition. For $n \geqslant 12$ and $1 \leqslant m \leqslant 1200$, the nonempty shells of norm $m$ of $\Gamma_{n}$ and of their shadows are not spherical 5-designs.

Proof. For $m<n / 4$, we remark that $\left(\Gamma_{n}\right)_{m}=\left(\mathbf{Z}^{n}\right)_{m}$ when $m$ is an even integer and $\left(\Gamma_{n}\right)_{m}=\emptyset$ otherwise. If moreover $n \equiv 4 \bmod 8$, then we have $\left(\mathcal{S h}\left(\Gamma_{n}\right)\right)_{m}=\left(\mathbf{Z}^{n}\right)_{m}$ when $m$ is an odd integer and $\left(\mathcal{S h}\left(\Gamma_{n}\right)\right)_{m}=\emptyset$ otherwise. Therefore, for $n>4800$, it suffices to apply Proposition 26 For $n \leqslant 4800$, we content ourself with a numerical verification.

The case of $\Gamma_{8}$ is considered in more details in the next Section.

\section{Even selfdual lattices of rank at most 24}

We recall here the classification of these lattices, due to Niemeier. Note that we already know by Proposition 3 that their rank is a multiple of 8 .

30. THEOREM.

(i) There is exactly one even selfdual lattice of rank 8, that is the KorkineZolotarev lattice

$$
\Gamma_{8}=\mathbf{E}_{8}^{+} .
$$

(ii) There are exactly two even selfdual lattices of rank 16, that is

$$
\Gamma_{8} \oplus \Gamma_{8}=\left(2 \mathbf{E}_{8}\right)^{+} \quad \text { and } \quad \Gamma_{16}=\mathbf{D}_{16}^{+} .
$$

(iii) There is a bijection between the even selfdual lattices of rank 24 and the twenty-four strongly eutactic root systems of norm 2 and of rank 24, given by $\Lambda \leftrightarrow \Lambda_{2}$. These root systems are (in parentheses is the Coxeter number of the system):

$$
\begin{array}{cccc}
\mathbf{O}_{24}(0), & 24 \mathbf{A}_{1}(2), & 12 \mathbf{A}_{2}(3), & 8 \mathbf{A}_{3}(4), \\
6 \mathbf{A}_{4}(5), & 4 \mathbf{A}_{5}+\mathbf{D}_{4}(6), & 6 \mathbf{D}_{4}(6), & 4 \mathbf{A}_{6}(7), \\
2 \mathbf{A}_{7}+2 \mathbf{D}_{5}(8), & 3 \mathbf{A}_{8}(9), & 9 \mathbf{A}_{9}+\mathbf{D}_{6}(10), & 4 \mathbf{D}_{6}(10), \\
\mathbf{A}_{11}+\mathbf{D}_{7}+\mathbf{E}_{6}(12), & 4 \mathbf{E}_{6}(12), & 2 \mathbf{A}_{12}(13), & 3 \mathbf{D}_{8}(14), \\
\mathbf{A}_{15}+\mathbf{D}_{9}(16), & \mathbf{D}_{10}+2 \mathbf{E}_{7}(18), & \mathbf{A}_{17}+\mathbf{E}_{7}(18), & 2 \mathbf{D}_{12}(22), \\
\mathbf{A}_{24}(25), & \mathbf{D}_{16}+\mathbf{E}_{8}(30), & 3 \mathbf{E}_{8}(30), & \mathbf{D}_{24}(46) .
\end{array}
$$

Claims (i) and (ii) are consequences of Claim (iii), since $\Lambda=R^{+}$is an even unimodular lattice of rank 8 [respectively 16] if and only if $\Lambda \oplus \Gamma_{8} \oplus \Gamma_{8}=\left(R+2 \mathbf{E}_{8}\right)^{+}$ [resp. $\left.\Lambda \oplus \Gamma_{8}=\left(R+\mathbf{E}_{8}\right)^{+}\right]$is an even unimodular lattice of rank 24 . 
The lattice corresponding to $\mathbf{O}_{24}$ is the famous Leech lattice, and those corresponding to nonempty strongly eutactic root systems of rank 24 are the the Niemeier lattices; those corresponding to the three last root systems listed are $\Gamma_{16} \oplus \Gamma_{8}$, $\Gamma_{8} \oplus \Gamma_{8} \oplus \Gamma_{8}$, and $\Gamma_{24}$ respectively.

There exist several proofs of Claim (iii); a proof explaining the bijection is found in Venk78.

\section{LEMma.}

(i) Let $\Lambda=\Gamma_{8}=\mathbf{E}_{8}^{+}$be the Korkine-Zolotareff lattice. Then

$$
\Theta_{\Lambda, P}= \begin{cases}\mathrm{Q} & \text { if } P=1, \\ 0 & \text { if } P \in \mathcal{H}^{(2 j)}\left(\mathbb{R}^{8}\right), 2 j=2,4,6,10, \\ c_{1}(P) \Delta_{24} & \text { if } P \in \mathcal{H}^{(8)}\left(\mathbb{R}^{8}\right), \\ c_{2}(P) \mathrm{Q} \Delta_{24} & \text { if } P \in \mathcal{H}^{(12)}\left(\mathbb{R}^{8}\right), \\ c_{3}(P) \mathrm{R} \Delta_{24} & \text { if } P \in \mathcal{H}^{(14)}\left(\mathbb{R}^{8}\right), \\ c_{4}(P) \mathrm{Q}^{2} \Delta_{24} & \text { if } P \in \mathcal{H}^{(16)}\left(\mathbb{R}^{8}\right), \\ c_{5}(P) \mathrm{R}^{2} \Delta_{24} & \text { if } P \in \mathcal{H}^{(18)}\left(\mathbb{R}^{8}\right),\end{cases}
$$

where $c_{1}, c_{2}, c_{3}, c_{4}$, and $c_{5}$ are nonzero linear form on $\mathcal{H}^{(8)}\left(\mathbb{R}^{8}\right), \mathcal{H}^{(12)}\left(\mathbb{R}^{8}\right)$, $\mathcal{H}^{(14)}\left(\mathbb{R}^{8}\right), \mathcal{H}^{(16)}\left(\mathbb{R}^{8}\right)$, and $\mathcal{H}^{(18)}\left(\mathbb{R}^{8}\right)$ respectively.

(ii) Let $\Lambda$ be one of the two even selfdual lattices of rank 16. Then

$$
\Theta_{\Lambda, P}= \begin{cases}\mathrm{Q}^{2} & \text { if } P=1, \\ 0 & \text { if } P \in \mathcal{H}^{(2 j)}\left(\mathbb{R}^{16}\right), 2 j=2,6, \\ c_{1}(P) \Delta_{24} & \text { if } P \in \mathcal{H}^{(4)}\left(\mathbb{R}^{16}\right), \\ c_{2}(P) \mathrm{Q} \Delta_{24} & \text { if } P \in \mathcal{H}^{(8)}\left(\mathbb{R}^{16}\right), \\ c_{3}(P) \mathrm{R} \Delta_{24} & \text { if } P \in \mathcal{H}^{(10)}\left(\mathbb{R}^{16}\right), \\ c_{4}(P) \mathrm{Q}^{2} \Delta_{24} & \text { if } P \in \mathcal{H}^{(12)}\left(\mathbb{R}^{16}\right), \\ c_{5}(P) \mathrm{RQ}^{2} \Delta_{24} & \text { if } P \in \mathcal{H}^{(14)}\left(\mathbb{R}^{16}\right),\end{cases}
$$

where $c_{1}, c_{2}, c_{3}, c_{4}$, and $c_{5}$ are nonzero linear forms on $\mathcal{H}^{(4)}\left(\mathbb{R}^{16}\right), \mathcal{H}^{(8)}\left(\mathbb{R}^{16}\right)$, $\mathcal{H}^{(10)}\left(\mathbb{R}^{16}\right), \mathcal{H}^{(12)}\left(\mathbb{R}^{16}\right)$, and $\mathcal{H}^{(14)}\left(\mathbb{R}^{16}\right)$ respectively.

(iii) Let $\Lambda$ be the Leech lattice. Then

$$
\Theta_{\Lambda, P}= \begin{cases}\mathrm{Q}^{3}-720 \Delta_{24} & \text { if } P=1, \\ 0 & \text { if } P \in \mathcal{H}^{(2 j)}\left(\mathbb{R}^{24}\right), 2 j=2,4,6,8,10,14, \\ c_{1}(P) \Delta_{24}^{2} & \text { if } P \in \mathcal{H}^{(12)}\left(\mathbb{R}^{24}\right), \\ c_{2}(P) \mathrm{Q} \Delta_{24}^{2} & \text { if } P \in \mathcal{H}^{(16)}\left(\mathbb{R}^{24}\right), \\ c_{3}(P) \mathrm{R} \Delta_{24}^{2} & \text { if } P \in \mathcal{H}^{(18)}\left(\mathbb{R}^{24}\right), \\ c_{4}(P) \mathrm{Q}^{2} \Delta_{24}^{2} & \text { if } P \in \mathcal{H}^{(20)}\left(\mathbb{R}^{24}\right), \\ c_{5}(P) \mathrm{RQ}^{2} \Delta_{24} & \text { if } P \in \mathcal{H}^{(22)}\left(\mathbb{R}^{24}\right),\end{cases}
$$

where $c_{1}, c_{2}, c_{3}, c_{4}$, and $c_{5}$ are nonzero linear forms on the corresponding spaces.

(iv) Let $\Lambda$ be an even selfdual lattice of rank 24 and of minimum 2. Let $h:=$ 
$\left|\Lambda_{2}\right| / 24$. Then

$$
\Theta_{\Lambda, P}= \begin{cases}\mathrm{Q}^{3}-24(30-h) \Delta_{24} & \text { if } P=1, \\ 0 & \text { if } P \in \mathcal{H}^{(2)}\left(\mathbb{R}^{n}\right), \\ c_{1}(P) \mathrm{Q} \Delta_{24} & \text { if } P \in \mathcal{H}^{(4)}\left(\mathbb{R}^{n}\right), \\ c_{2}(P) \mathrm{R} \Delta_{24} & \text { if } P \in \mathcal{H}^{(6)}\left(\mathbb{R}^{n}\right), \\ c_{3}(P) \mathrm{Q}^{2} \Delta_{24} & \text { if } P \in \mathcal{H}^{(8)}\left(\mathbb{R}^{n}\right), \\ c_{4}(P) \mathrm{RQ}^{2} \Delta_{24} & \text { if } P \in \mathcal{H}^{(10)}\left(\mathbb{R}^{n}\right),\end{cases}
$$

where $c_{1}, c_{2}, c_{3}$, and $c_{4}$ are nonzero linear forms on the corresponding spaces.

Proof. It suffices to apply Propositions [15] and 16] In order to see that the linear forms $c_{i}, i=1,2, \ldots$, are nonzero, we apply Lemma 5 and Proposition 23] to $\Lambda_{2}$ if $\Lambda$ is not the Leech lattice, and to $\Lambda_{4}$ if $\Lambda$ is the Leech lattice. (Note that, since $\left\{\langle x \mid y\rangle \mid x, y \in \Lambda_{4}\right\}=\{0, \pm 1, \pm 2, \pm 4\}$, the shell $\Lambda_{4}$ is a tight 11-design: see DeGoSe77, Theorems 5.12 and 6.8] or [GoeSei79, Theorems 5.3 and 5.4]. It follows that $\Lambda_{4}$ cannot be a 12-design.)

Remark. In fact, Propositions [15] and [16] show that the root system of an even selfdual lattice of rank 8 [respectively 16,24$]$ is nonempty and a $7^{1} / 2^{-d e s i g n}$ [resp. is nonempty and a $5^{1} / 2^{-}$design, is strongly eutactic]. Theorem [30 says that, for each root system in dimension up to 24 which satisfies the above condition, there exists exactly one even selfdual lattice with such a root system. But in higher dimensions, there can be more than one even selfdual lattice with the same root system.

As in the preceding case, Lemma $[$ give:

\section{THEOREM.}

(i) All shells of $\Gamma_{8}=\mathbf{E}_{8}^{+}$are spherical $7^{1} / 2^{-d e s i g n s . ~}$

(ii) All shells of the two even selfdual lattices of rank 16 are spherical $3^{1} / 2^{-d e s i g n s . ~}$

(iii) All shells of the Leech lattice are spherical 11/ $/ 2$-designs.

(iv) All shells of any even selfdual lattice of rank 24 and of minimum 2 are spherical 3-designs.

Remark. We have checked numerically that the shells of norm at most 1200 of these lattices are not spherical designs of higher strength.

Claims (i) to (iii) of the Theorem above are special cases of Theorem 16.4 of VenMar01 (see also Venk84), since the lattices mentioned in these claims are extremal. See the remark at the end of Section 5

Another consequence of our analysis is a reformulation in terms of spherical design strength of shells of lattices of a famous conjecture of Lehmer, which states that the Ramanujan numbers $\tau(m)$ are never zero for $m \geqslant 1$. This conjecture has been verified for $m \leqslant 10^{15}$ Serr85, $\left.\S 3.3\right]$.

33. Proposition. Let $\tau(m), m \geqslant 1$, be the Ramanujan numbers defined by

$$
\Delta_{24}(z)=\sum_{m \geqslant 1} \tau(m) q^{2 m} .
$$

For $m \geqslant 1$, the following are equivalent:

(a) $\tau(m)=0$; 
(b) $\left(\Gamma_{8}\right)_{2 m}$ is an 8-design (and therefore an 11-design);

(c) for any even selfdual lattice $\Lambda$ of rank 16, $\Lambda_{2 m}$ is a 4-design (and therefore a 7-design);

Therefore, Lehmer's conjecture is true if and only if no shell of $\Gamma_{8}$ is an 8-design, and if and only if no shell of any even selfdual lattice of rank 16 is a 4-design.

Similar conjectures could be stated for other modular forms than $\Delta_{24}$, and have an equivalent formulation in terms of spherical design strength of shells of lattices (see Proposition $\mathrm{C}$ in the Introduction).

\section{Selfdual lattices with long shadow}

In this section, we consider selfdual lattices with $\sigma(\Lambda)=n-8$. We begin with those of minimum at least 2. We recall here their classification Elki95b.

34. Theorem. There is a bijection between the selfdual lattices with $\sigma(\Lambda)=$ $n-8$ of minimum at least 2 and the 14 strongly eutactic root systems satisfying $h=2(23-n)$, given by $\Lambda \leftrightarrow \Lambda_{2}$. These root systems are (in parentheses are the corresponding values of $n$ and $h$ ):

$$
\begin{array}{cccc}
\mathbf{E}_{8}(8,30), \quad \mathbf{D}_{12} & (12,26), & 2 \mathbf{E}_{7}(14,18), & \mathbf{A}_{15}(15,16), \\
2 \mathbf{D}_{8}(16,14), & \mathbf{A}_{11}+\mathbf{E}_{6}(17,12), & 2 \mathbf{A}_{9}(18,10), \\
3 \mathbf{D}_{6}(18,10), & 2 \mathbf{A}_{7}+\mathbf{D}_{5}(19,8), & 4 \mathbf{A}_{5}(20,6), \\
5 \mathbf{D}_{4}(20,6), \quad 7 \mathbf{A}_{3}(21,4), & 22 \mathbf{A}_{1}(22,2), & \mathbf{O}_{23}(23,0) .
\end{array}
$$

Recall that we denote by $R^{+}$the (unique up to dimension 23) selfdual lattice $\Lambda$ of root system $R=\Lambda_{2}$. Apart from $\Gamma_{8}=\mathbf{E}_{8}^{+}$, which is even, all these lattices are odd. They are of minimum 2, except $\mathbf{O}_{23}^{+}$which is of minimum 3 (it is the so-called shorter Leech lattice).

The theta series of such lattices are now easy to calculate (we exclude here the theta series of $\Gamma_{8}=\mathbf{E}_{8}^{+}$, which have been given in Lemma 31).

35. Lemma. Let $\Lambda$ be an odd selfdual lattice with $\sigma(\Lambda)=n-8$ and of minimum at least 2 .

(i) If $\Lambda$ is of minimum 2 , then

$$
\Theta_{\Lambda, P}= \begin{cases}\theta_{3}^{n}-2 n \theta_{3}^{n-8} \Delta_{8} & \text { if } P=1, \\ 0 & \text { if } P \in \mathcal{H}^{(2)}\left(\mathbb{R}^{n}\right), \\ c_{1}(P) \theta_{3}^{n-8} \Delta_{8}^{2} & \text { if } P \in \mathcal{H}^{(4)}\left(\mathbb{R}^{n}\right), \\ c_{2}(P) \Phi \theta_{3}^{n-8} \Delta_{8}^{2} & \text { if } P \in \mathcal{H}^{(6)}\left(\mathbb{R}^{n}\right) .\end{cases}
$$

where $c_{1}$ and $c_{2}$ are nonzero linear forms on $\mathcal{H}^{(4)}\left(\mathbb{R}^{n}\right)$ and $\mathcal{H}^{(6)}\left(\mathbb{R}^{n}\right)$ respectively.

(ii) If $\Lambda=\mathbf{O}_{23}^{+}$is the shorter Leech lattice, then

$$
\Theta_{\Lambda, P}= \begin{cases}\theta_{3}^{23}-46 \theta_{3}^{15} \Delta_{8} & \text { if } P=1 \\ 0 & \text { if } P \in \mathcal{H}^{(2 j)}\left(\mathbb{R}^{23}\right), 2 j=2,4,6, \\ c_{1}(P) \theta_{3}^{15} \Delta_{8}^{3} & \text { if } P \in \mathcal{H}^{(8)}\left(\mathbb{R}^{23}\right), \\ c_{2}(P) \Phi \theta_{3}^{15} \Delta_{8}^{3} & \text { if } P \in \mathcal{H}^{(10)}\left(\mathbb{R}^{23}\right),\end{cases}
$$

where $c_{1}$ and $c_{2}$ are nonzero linear forms on $\mathcal{H}^{(8)}\left(\mathbb{R}^{n}\right)$ and $\mathcal{H}^{(10)}\left(\mathbb{R}^{n}\right)$ respectively. 
Proof. Propositions 13 and 14 give the forms of the theta series (incidently, they show that $\Lambda_{2}$ is strongly eutactic and that $h=2(23-n)$ as stated in Theorem 34), except the fact that the linear forms $c_{1}$ and $c_{2}$ are nonzero.

If $\Lambda$ is of minimum 2, by Proposition 5 applied to the root system of $\Lambda$, the linear forms $c_{1}$ and $c_{2}$ are nonzero.

Consider now the case $\Lambda=\mathbf{O}_{23}^{+}$. Let $x \in \Lambda_{3}$, and set $N_{\alpha}:=\mid\left\{y \in \Lambda_{3} \mid\langle x \mid y\rangle=\right.$ $\alpha\} \mid$. It is known that

$$
N_{3}=N_{-3}=2, \quad N_{1}=N_{-1}=891, \quad N_{0}=2816,
$$

and $N_{\alpha}=0$ for other $\alpha$ 's. (This follows from he fact that $\Lambda_{3}$ is a tight 7-design: see DeGoSe77, Theorems 5.12 and 6.8] or GoeSei79, Theorems 5.3 and 5.4].) From this, using Lemma 22 (with $\lambda_{x}=1$ if $x \in \Lambda_{3}$, and $\lambda_{x}=0$ otherwise), it is easy to see that $c_{1}$ and $c_{2}$ are indeed nonzero.

The zero coefficients of the Fourier series for the modular forms of the Lemma are given by Lemma [17] We deduce:

36. THEOREM.

(i) Every nonempty shell of any selfdual lattice with $\sigma(\Lambda)=n-8$ of minimum 2, and every nonempty shell of its shadow, is a spherical 3-design.

(ii) The following shells are spherical $3^{1} / 2^{\text {-designs: }}$

$$
\left(2 \mathbf{D}_{8}\right)^{+}: \quad m=4^{a}, a \geqslant 1
$$

(iii) The following shells are spherical 5-designs:

$$
\begin{array}{ll}
\left(4 \mathbf{A}_{5}\right)^{+},\left(5 \mathbf{D}_{4}\right)^{+}: m & =4^{a}, a \geqslant 1 \\
\left(2 \mathbf{D}_{8}\right)^{+}: & m=2 a+1, a \geqslant 1
\end{array}
$$

(iv) All nonempty shells of the shorter Leech lattice $\mathbf{O}_{23}^{+}$and of its shadow are spherical 7-designs.

Remark. We have checked numerically that the shells of norm at most 1200 of these lattices and of their shadows are not spherical designs of higher strength.

Let us now turn to selfdual lattices of minimum 1.

37. Lemma. Let $\Lambda$ be a selfdual lattice with $\sigma(\Lambda)=n-8$ of minimum 1 .

(i) If $\Lambda=\mathbf{Z}^{1} \oplus \mathbf{E}_{8}^{+}=\mathbf{Z}^{1} \oplus \Gamma_{8}$, then

$$
\Theta_{\Lambda, P}= \begin{cases}\theta_{3}^{9}-16 \theta_{3} \Delta_{8} & \text { if } P=1, \\ c_{1}(P) \Phi \theta_{3} \Delta_{8} & \text { if } P \in \mathcal{H}^{(2)}\left(\mathbb{R}^{9}\right), \\ c_{2}(P)\left(\theta_{3}^{9} \Delta_{8}+8 \theta_{3} \Delta_{8}^{2}\right) & \text { if } P \in \mathcal{H}^{(4)}\left(\mathbb{R}^{9}\right), \\ c_{4}(P)\left(\Phi \theta_{3}^{9} \Delta_{8}-\Phi \theta_{3} \Delta_{8}^{2}\right) & \text { if } P \in \mathcal{H}^{(6)}\left(\mathbb{R}^{9}\right),\end{cases}
$$

where $c_{1}, c_{2}$ and $c_{4}$ are nonzero linear forms on $\mathcal{H}^{(2)}\left(\mathbb{R}^{9}\right), \mathcal{H}^{(4)}\left(\mathbb{R}^{9}\right)$ and $\mathcal{H}^{(6)}\left(\mathbb{R}^{9}\right)$ respectively. 
(ii) If $\Lambda=\mathbf{Z}^{1} \oplus \mathbf{O}_{23}^{+}$, then

$$
\Theta_{\Lambda, P}= \begin{cases}\theta_{3}^{24}-46 \theta_{3}^{16} \Delta_{8} & \text { if } P=1, \\ c_{1}(P) \Phi \theta_{3}^{16} \Delta_{8} & \text { if } P \in \mathcal{H}^{(2)}\left(\mathbb{R}^{24}\right), \\ c_{2}(P)\left(\theta_{3}^{24} \Delta_{8}-40 \theta_{3}^{16} \Delta_{8}^{2}\right) & \text { if } P \in \mathcal{H}^{(4)}\left(\mathbb{R}^{24}\right), \\ c_{4}(P)\left(\Phi \theta_{3}^{24} \Delta_{8}-16 \Phi \theta_{3}^{16} \Delta_{8}^{2}\right) & \text { if } P \in \mathcal{H}^{(6)}\left(\mathbb{R}^{24}\right),\end{cases}
$$

where $c_{1}, c_{2}$ and $c_{4}$ are nonzero linear forms on $\mathcal{H}^{(2)}\left(\mathbb{R}^{24}\right), \mathcal{H}^{(4)}\left(\mathbb{R}^{24}\right)$ and $\mathcal{H}^{(6)}\left(\mathbb{R}^{24}\right)$ respectively.

(iii) Otherwise, we have

$$
\Theta_{\Lambda, P}= \begin{cases}\theta_{3}^{n}-2 n \theta_{3}^{n-8} \Delta_{8} & \text { if } P=1, \\ c_{1}(P) \Phi \theta_{3}^{n-8} \Delta_{8} & \text { if } P \in \mathcal{H}^{(2)}\left(\mathbb{R}^{n}\right), \\ c_{2}(P) \theta_{3}^{n} \Delta_{8}+c_{3}(P) \theta_{3}^{n-8} \Delta_{8}^{2} & \text { if } P \in \mathcal{H}^{(4)}\left(\mathbb{R}^{n}\right), \\ c_{4}(P) \Phi \theta_{3}^{n} \Delta_{8}+c_{5}(P) \Phi \theta_{3}^{n-8} \Delta_{8}^{2} & \text { if } P \in \mathcal{H}^{(6)}\left(\mathbb{R}^{n}\right),\end{cases}
$$

where $c_{1}$ is a nonzero linear form on $\mathcal{H}^{(2)}\left(\mathbb{R}^{n}\right), c_{2}$ and $c_{3}$ are linearly independent linear forms on $\mathcal{H}^{(4)}\left(\mathbb{R}^{n}\right)$, and $c_{4}$ and $c_{5}$ are linearly independent linear forms on $\mathcal{H}^{(6)}\left(\mathbb{R}^{n}\right)$.

Proof. Let $\Lambda=\mathbf{Z}^{p} \oplus L$, where $L$ is a lattice of minimum 2 and of rank $N$, and let $h$ be the Coxeter number of the strongly eutactic root lattice $L_{2}$. Let $V_{1}$ [respectively $V_{2}$ ] be the space generated by $\mathbf{Z}^{p}$ [resp. $L$ ], so that $\mathbb{R}^{n}=V_{1} \oplus V_{2}$. For $x \in \mathbb{R}^{n}$, let $x_{i} \in V_{i}(i=1,2)$ such that $x=x_{1}+x_{2}$. Let $\omega_{i}(x):=\left\langle x_{i} \mid x_{i}\right\rangle$.

First, Proposition 13 gives the exact form of $\Theta_{\Lambda}$.

Now, by Proposition [14 we have $\Theta_{\Lambda, P}=c_{1}(P) \Phi \theta_{3}^{n-8} \Delta_{8}$ for $P \in \mathcal{H}^{(2)}\left(\mathbb{R}^{n}\right)$. The polynomial

$$
P:=\frac{1}{2 p} \omega_{1}-\frac{1}{2 N} \omega_{2} \in \mathcal{H}^{(2)}\left(\mathbb{R}^{n}\right)
$$

verifies $\Theta_{\Lambda, P}(z)=q+O\left(q^{2}\right) \neq 0$; thus $c_{1}$ is not identically equal to zero.

Then, we have $\Theta_{\Lambda, P}=c_{2}(P) \theta_{3}^{n} \Delta_{8}+c_{3}(P) \theta_{3}^{n-8} \Delta_{8}^{2}$ for $P \in \mathcal{H}^{(4)}\left(\mathbb{R}^{n}\right)$. Let be the polynomial

$$
Q:=\frac{1}{2 p} \omega_{1}^{2}-\frac{p+2}{p N} \omega_{1} \omega_{2}+\frac{p+2}{2 N(N+2)} \omega_{2}^{2} \in \mathcal{H}^{(4)}\left(\mathbb{R}^{n}\right) .
$$

We have

$$
\begin{aligned}
\Theta_{\Lambda, Q}(z) & =q+4\left((p-1)+\frac{h(p+2)}{2(N+2)}\right) q^{2}+O\left(q^{3}\right) \\
& =\left(q+(2 n-8) q^{2}+O\left(q^{3}\right)\right)+\left(\left(2 p-2 N+4+\frac{2 h(p+2)}{N+2}\right) q^{2}+O\left(q^{3}\right)\right) \\
& =\theta_{3}^{n}(z) \Delta_{8}(z)+\left(2 p-2 N+4+\frac{2 h(p+2)}{N+2}\right) \theta_{3}^{n-8}(z) \Delta_{8}^{2}(z) .
\end{aligned}
$$

If $L$ is neither $\mathbf{O}_{23}^{+}$nor $\mathbf{E}_{8}^{+}$, then, by Lemma 35 there exists a $R \in \mathcal{H}^{(4)}\left(V_{2}\right) \subseteq$ $\mathcal{H}^{(4)}\left(\mathbb{R}^{n}\right)$ such that $\Theta_{L, R} \neq 0$. So we have

$$
\Theta_{\Lambda, R}(z)=\Theta_{\mathbf{Z}^{p}, 1}(z) \Theta_{L, R}(z)=c_{4}(R) q^{2}+O\left(q^{3}\right)=c_{4}(R) \theta_{3}^{n-8}(z) \Delta_{8}^{2}(z),
$$

with $c_{4}(R) \neq 0$; that shows that $c_{3}$ and $c_{4}$ are linearly independant.

If $\Lambda=\mathbf{Z}^{p} \oplus \mathbf{E}_{8}^{+}$and if $p \geqslant 2$, we can find a $S \in \mathcal{H}^{(4)}\left(V_{1}\right) \subseteq \mathcal{H}^{(4)}\left(\mathbb{R}^{n}\right)$ such that $\Theta_{\mathbf{Z}^{p}, S}=\theta_{3}^{p} \Delta_{8}$. So we have

$$
\Theta_{\mathbf{Z}^{p} \oplus \mathbf{E}_{8}^{+}, S}=\Theta_{\mathbf{Z}^{p}, S} \Theta_{\mathbf{E}_{8}^{+}, 1}=\theta_{3}^{p} \Delta_{8}\left(\theta_{3}^{8}-16 \Delta_{8}\right)=\theta_{3}^{n} \Delta_{8}-16 \theta_{3}^{n-8} \Delta_{8} .
$$


Therefore, if we compare

$$
\begin{gathered}
\Theta_{\mathbf{Z}^{p} \oplus \mathbf{E}_{8}^{+}, Q}=\theta_{3}^{n}(z) \Delta_{8}(z)+8 p \theta_{3}^{n-8}(z) \Delta_{8}^{2}(z) ., \\
\Theta_{\mathbf{Z}^{p} \oplus \mathbf{E}_{8}^{+}, S}=\theta_{3}^{n} \Delta_{8}-16 \theta_{3}^{n-8} \Delta_{8}
\end{gathered}
$$

we find that $c_{2}$ and $c_{3}$ are linearly independent.

If $\Lambda=\mathbf{Z}^{1} \oplus \mathbf{E}_{8}^{+}$, it can be shown that $c_{2}$ and $c_{3}$ are not linearly independent. We do not prove it, since it has no effect on the conclusions of the following Theorem.

If $\Lambda=\mathbf{Z}^{p} \oplus \mathbf{O}_{23}^{+}$, we proceed as for $\mathbf{Z}^{p} \oplus \mathbf{E}_{8}^{+}$.

A similar method is used for computing $\Theta_{\Lambda, P}$ for $P \in \mathcal{H}^{(6)}\left(\mathbb{R}^{n}\right)$. Note however that in the cases $\mathbf{Z}^{2} \oplus \mathbf{E}_{8}^{+}$and $\mathbf{Z}^{2} \oplus \mathbf{E}_{8}^{+}$, the polynomial corresponding to $S$ is a polynomial of the form

$$
S^{\prime}=\left(N \omega_{1}-(p+8) \omega_{2}\right) f \in \mathcal{H}^{(6)}\left(\mathbb{R}^{n}\right), \quad \text { where } f \in \mathcal{H}^{(4)}\left(V_{1}\right) .
$$

and such that $\Theta_{\mathbf{Z}^{2}, f} \neq 0$, since $\Theta_{\mathbf{Z}^{2}, P}=0$ for every $P \in \mathcal{H}^{(6)}\left(V_{1}\right)$.

Again, Lemma 17 describes indices of vanishing coefficients for the series of the previous Lemma. Therefore, we have:

\section{THEOREM.}

(i) Let $\Lambda$ be a selfdual lattice of rank 24 with $\sigma(\Lambda)=24-8=16$. Then the shells $\Lambda_{m}$ and $\mathcal{S h}(\Lambda)_{m}$ are spherical 3-designs for $m=4^{a} 2, a \geqslant 0$ (except the shells $\mathcal{S h}(\Lambda)_{2}$ and $\left(\mathbf{Z}^{1} \oplus \mathbf{O}_{23}^{+}\right)_{2}$, which are empty).

(ii) Let $\Lambda=\mathbf{Z}^{3} \oplus \Gamma_{8}$. Then the shells $\Lambda_{m}$ are spherical 3-designs for $m=4^{a}(8 b+7)$, $a, b \geqslant 0$.

Remark. We have checked numerically that the shells of norm at most 1200 of these lattices and of their shadows are not spherical designs of higher strength.

\section{Odd selfdual lattices of rank 24 and of minimum at least 2}

We need to know the precise form of the shell of norm 2 of these lattices and their shadows:

39. Proposition. Let $\Lambda$ be an odd selfdual lattice of rank 24 and of minimum 2. Then there exist a root system $R$, and strongly eutactic root systems $S$ and $T$ of Coxeter number $h_{S}$ and $h_{T}$ respectively, such that

$-R=S \cap T$,

- $h_{S}+h_{T}=3 h_{R}+2$ where $h_{R}:=|R| / 24$,

$-s \in S \backslash R, t \in T \backslash R \Longrightarrow\langle s \mid t\rangle= \pm \frac{1}{2}$,

- $\Lambda_{2}=R$, and $\mathcal{S h}(\Lambda)_{2}=(S \backslash R) \cup(T \backslash R)$.

Moreover, $T=R$ or $S=R$ if and only if $R$ is strongly eutactic.

Sketch of the proof. Let $\Lambda$ be an odd selfdual lattice of rank 24 and of minimum 2 . Let $\Lambda_{0}$ be the even sublattice of index 2 , and let $\Lambda_{0}^{\sharp}$ be its dual. Then $\Lambda_{0}^{\sharp} / \Lambda_{0} \simeq$ 
$\mathbb{Z} / 2 \mathbb{Z} \oplus \mathbb{Z} / 2 \mathbb{Z}$, and we have the following diagramm (integers indicate indices of sublattices):

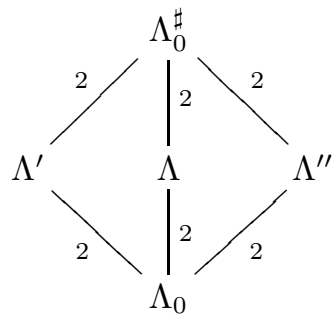

where $\Lambda^{\prime}$ and $\Lambda^{\prime \prime}$ are even selfdual lattices. We have $\mathcal{S h}(\Lambda)=\left(\Lambda^{\prime} \backslash \Lambda_{0}\right) \cup\left(\Lambda^{\prime \prime} \backslash \Lambda_{0}\right)$. The root systems of the Proposition are

$$
R=\Lambda_{2}, \quad S=\Lambda_{2}^{\prime}, \quad T=\Lambda_{2}^{\prime \prime},
$$

and, according to Theorem 30 iii, $S$ and $T$ are strongly eutactic. By Proposition 13 the theta series of $\Lambda$ is

$$
\Theta_{\Lambda}=\theta_{3}^{n}+c_{1} \Delta_{8} \theta_{3}^{n-8}+c_{2} \Delta_{8}^{2} \theta_{3}^{n-16},
$$

where

$$
\begin{aligned}
c_{2} & =24\left(\left|\Lambda_{2}\right| / 24-2\right)=24\left(h_{R}-2\right) \\
& =\left|\operatorname{Sh}(\Lambda)_{2}\right|=24\left(h_{S}+h_{T}-2 h_{R}\right),
\end{aligned}
$$

hence $h_{S}+h_{T}=3 h_{R}+2$.

For $x \in \mathbb{R}^{24}$ and $\alpha \in \mathbb{R}$, let $N_{\alpha}^{x, A}:=|\{y \in A \mid\langle x \mid y\rangle=\alpha\}|$. Let $s \in S \backslash R$; it can be shown that (see Lemma 40 below):

$$
\begin{gathered}
N_{1}^{s, R}=N_{-1}^{s, R}=N_{1}^{s, T}=N_{-1}^{s, T}=3 h_{R}-h_{T}=h_{S}-2, \\
N_{1 / 2}^{s, T}=N_{-1 / 2}^{s, T}=12\left(h_{T}-h_{R}\right), \\
N_{0}^{s, R}=N_{0}^{s, T}=8 h_{T}+6 h_{S}-12 .
\end{gathered}
$$

Let us suppose that $R$ is strongly eutactic and that $S \backslash R \neq \emptyset$. Let $s \in S \backslash R$; since the polynomial function $x \mapsto\langle s \mid x\rangle^{2}-2\langle x \mid x\rangle$ is harmonic, we have the equality

$$
\sum_{r \in R}\langle s \mid r\rangle^{2}=4 h_{R}
$$

hence

$$
2 h_{S}-4=N_{1}^{s, R}+N_{-1}^{s, R}=\sum_{r \in R}\langle s \mid r\rangle^{2}=4 h_{R} .
$$

Using the equality $h_{S}+h_{T}=3 h_{R}+2$, we deduce that $h_{T}=h_{R}$, therefore $T=R$ and $h_{S}=2 h_{R}+2$. Conversely, if $T=R$, then $R$ is strongly eutactic.

To complete the proof, we establish formulae $(*)$ above:

40. Lemma. In the proof of the preceding Lemma, formulae $(*)$ hold.

Sketch of the proof. Let $s \in S \backslash R$. The scalar product on $\Lambda_{0}^{\sharp}$ reduces to a bilinear form on $\Lambda_{0}^{\sharp} / \Lambda_{0}$ with values in $\left(\frac{1}{2} \mathbb{Z}\right) / \mathbb{Z}$, which is easy to explicit. In particular, for $t \in T$, we have:

$$
\langle s \mid t\rangle= \begin{cases} \pm 1 / 2 & \text { if } t \in T \backslash R \\ 0, \pm 1 & \text { if } t \in R .\end{cases}
$$


(The values $\pm 3 / 2$ are excluded in the first case by computing the norm of $s \mp t$.) From this, we deduce $N_{ \pm 1 / 2}^{s, T}=12\left(h_{T}-h_{R}\right)$, and $N_{\epsilon}^{s, R}=N_{\epsilon}^{s, T}$ for $\epsilon=0, \pm 1$. Finally, $N_{\epsilon}^{s, T}$ is deduced from the strongly eutaxy of $T$. For example, we have

$$
-\frac{1}{4} N_{-1 / 2}^{s, T}+\frac{3}{4} N_{1 / 2}^{s, T}+2 N_{1}^{s, T}=\sum_{t \in T}(\langle s \mid t\rangle+1)\langle s \mid t\rangle=2 h_{T} .
$$

The last equality follows from the fact that $T$ is a spherical 2-design (use Theorem 3.2 and Formula 3.6 in VenMar01, or Proposition 1.13 in HarPac04b). Therefore $N_{1}^{s, T}=3 h_{R}-h_{T}$.

There are 156 odd selfdual lattices of rank 24, classified in Borc84 and listed in BorcCS. We give here the list of those with strongly eutactic root system, because it is the occasion to point out a remarkable bijection between two sets (compare with Theorems [30]iii and 34).

41. Theorem. There is a bijection between the set of odd selfdual lattices $\Lambda$ of rank 24, of minimum 2, and of strongly eutactic root system, and the set of pairs of embedded strongly eutactic root systems $R \subseteq S$ in $\mathbb{R}^{24}$ satisfying $h_{S}=2 h_{R}+2$. The bijection is given by $R=\Lambda_{2}$ and $S=\mathcal{S h}(\Lambda)_{2}$. The list of pairs $R \subseteq S$ is given below. (The numbers in brackets refer to Table 17.1 of BorcCS $^{1}$ and Table III of Bach97. These lattices correspond to bold edges in the neighbourhood graph of Figure 17.1 in BorcCS].)

$$
\mathbf{O}_{24} \subseteq 24 \mathbf{A}_{1}[1], \quad 24 \mathbf{A}_{1} \subseteq 6 \mathbf{D}_{4}[6], \quad 8 \mathbf{A}_{3} \subseteq 4 \mathbf{D}_{6}[32]
$$

$6 \mathbf{D}_{4} \subseteq 3 \mathbf{D}_{8}$ [74], $\quad 2 \mathbf{A}_{7}+\mathbf{D}_{5} \subseteq \mathbf{D}_{10}+2 \mathbf{E}_{7}$ [105], $\quad 4 \mathbf{D}_{6} \subseteq 2 \mathbf{D}_{12}$ [130],

$$
3 \mathbf{D}_{8} \subseteq 3 \mathbf{E}_{8}[145], \quad 3 \mathbf{D}_{8} \subseteq \mathbf{E}_{8}+\mathbf{D}_{16}[146], \quad 2 \mathbf{D}_{12} \subseteq \mathbf{D}_{24}[154] .
$$

We are now ready to give the theta series for selfdual lattices of rank 24 :

42. Lemma. Let $\Lambda$ be an odd selfdual lattice of rank 24, and of minimum at least 2 .

(i) If $\Lambda_{2}$ is not strongly eutactic, and if $h:=\left|\Lambda_{2}\right| / 24$, then

$$
\Theta_{\Lambda, P}= \begin{cases}\theta_{3}^{24}-48 \theta_{3}^{16} \Delta_{8}+24(h+2) \theta_{3}^{8} \Delta_{8}^{2} & \text { if } P=1, \\ c_{1}(P) \Phi \theta_{3}^{8} \Delta_{8}^{2} & \text { if } P \in \mathcal{H}^{(2)}\left(\mathbb{R}^{24}\right), \\ c_{2}(P) \theta_{3}^{16} \Delta_{8}^{2}+c_{3}(P) \theta_{3}^{8} \Delta_{8}^{3} & \text { if } P \in \mathcal{H}^{(4)}\left(\mathbb{R}^{24}\right),\end{cases}
$$

where $c_{1}$ is a nonzero linear form on $\mathcal{H}^{(2)}\left(\mathbb{R}^{24}\right)$, and $c_{2}$ and $c_{3}$ are linearly independent linear forms on $\mathcal{H}^{(4)}\left(\mathbb{R}^{24}\right)$.

(ii) If $\Lambda_{2}$ is nonempty and strongly eutactic of Coxeter number $h$, then

$$
\Theta_{\Lambda, P}= \begin{cases}\theta_{3}^{24}-48 \theta_{3}^{16} \Delta_{8}+24(h+2) \theta_{3}^{8} \Delta_{8}^{2} & \text { if } P=1, \\ 0 & \text { if } P \in \mathcal{H}^{(2)}\left(\mathbb{R}^{24}\right), \\ c_{2}(P) \theta_{3}^{16} \Delta_{8}^{2}+c_{3}(P) \theta_{3}^{8} \Delta_{8}^{3} & \text { if } P \in \mathcal{H}^{(4)}\left(\mathbb{R}^{24}\right), \\ c_{4}(P) \Phi \theta_{3}^{16} \Delta_{8}^{2}+c_{5}(P) \Phi \theta_{3}^{8} \Delta_{8}^{3} & \text { if } P \in \mathcal{H}^{(6)}\left(\mathbb{R}^{24}\right),\end{cases}
$$

where $c_{2}$ and $c_{3}$ are linearly independent linear forms on $\mathcal{H}^{(4)}\left(\mathbb{R}^{24}\right)$, and $c_{4}$ and $c_{5}$ are linearly independent linear forms on $\mathcal{H}^{(6)}\left(\mathbb{R}^{24}\right)$.

\footnotetext{
${ }^{1}$ There are some errors in that table in the two first editions, which have been corrected in the third edition.
} 
(iii) If $\Lambda_{2}$ is empty, then

$$
\Theta_{\Lambda, P}= \begin{cases}\theta_{3}^{24}-48 \theta_{3}^{16} \Delta_{8}+48 \theta_{3}^{8} \Delta_{8}^{2} & \text { if } P=1, \\ 0 & \text { if } P \in \mathcal{H}^{(2)}\left(\mathbb{R}^{24}\right), \\ c_{3}(P) \theta_{3}^{8} \Delta_{8}^{3} & \text { if } P \in \mathcal{H}^{(4)}\left(\mathbb{R}^{24}\right), \\ c_{5}(P) \Phi \theta_{3}^{8} \Delta_{8}^{3} & \text { if } P \in \mathcal{H}^{(6)}\left(\mathbb{R}^{24}\right),\end{cases}
$$

where $c_{3}$ and $c_{5}$ are nonzero linear forms on $\mathcal{H}^{(4)}\left(\mathbb{R}^{24}\right)$ and $\mathcal{H}^{(6)}\left(\mathbb{R}^{24}\right)$ respectively.

Idea of the proof. We use Proposition 13 for computing the theta series. The main difficulty is to show that, for example, in case (ii), the coefficients $c_{2}$ and $c_{3}$ are linearly independant. (Similar cases are treated in the same way.) The main idea is to look at the theta series of the shadow (Proposition 10):

$$
\begin{gathered}
\Theta_{\Lambda, P}=c_{2}(P) \theta_{3}^{16} \Delta_{8}^{2}+c_{3}(P) \theta_{3}^{8} \Delta_{8}^{3}=c_{2}(P) q^{2}+O\left(q^{3}\right), \\
\Theta_{\mathcal{S} h(\Lambda), P}=c_{2}(P) \theta_{2}^{16} \mathcal{S} h \Delta_{8}^{2}+c_{3}(P) \theta_{2}^{8} \operatorname{Sh} \Delta_{8}^{3}=-2^{4} c_{3}(P) q^{2}+O\left(q^{4}\right) .
\end{gathered}
$$

So, we have

$$
c_{2}(P)=\sum_{x \in \Lambda_{2}} P(x) \quad \text { and } \quad c_{3}(P)=-2^{-4} \sum_{x \in \mathcal{S h}(\Lambda)_{2}} P(x) .
$$

The shape of $\Lambda_{2}$ and $\mathcal{S h}(\Lambda)_{2}$ is given by Proposition [39] Finally, one can find two harmonic homogeneous polynomials of degree 4 which give linearly independent values of $c_{2}(P)$ and $c_{3}(P)$.

We can now conclude:

43. Theorem. Let $\Lambda$ be an odd selfdual lattice of rank 24 and of minimum 2 .

(i) If $\Lambda_{2}$ is strongly eutactic, then all nonnempty shells of $\Lambda$ and $\mathcal{S h}(\Lambda)$ are 3designs.

(ii) If $\Lambda_{2}$ is not strongly eutactic, then the shells $\Lambda_{m}$ and $\mathcal{S h}(\Lambda)_{m}$ are 3-designs for $m=4^{a}, a \geqslant 1$.

Remark. We have checked numerically that the shells of norm at most 1200 of these lattices and of their shadows are not spherical designs of higher strength.

\section{Other selfdual lattices up to rank 24}

According to their classification ([ConSlo99, Chap. 16 and 17] and [Bach97]), the remaining selfdual lattices of rank at most 24 enter in one of the three cases of the following Lemma:

44. Lemma. Let $\Lambda=\mathbf{Z}^{p} \oplus L \subseteq \mathbb{R}^{n}$ be a selfdual lattice with $\sigma(\Lambda)=n-16$, where $L \subseteq \mathbb{R}^{N}$ is of minimum 2 .

(i) If $p=0$ and if $L_{2}$ is not strongly eutactic, then

$$
\Theta_{\Lambda, P}=c(P) \Phi \theta_{3}^{n-16} \Delta_{8}^{2} \quad \text { if } P \in \mathcal{H}^{(2)}\left(\mathbb{R}^{n}\right),
$$

where $c$ is a nonzero linear form on $\mathcal{H}^{(2)}\left(\mathbb{R}^{n}\right)$. 
(ii) If $p \geqslant 1$ and if $L_{2}$ is strongly eutactic of Coxeter number $h$, then

$$
\Theta_{\Lambda, P}=c(P)\left(\Phi \theta_{3}^{n-8} \Delta_{8}+(46-2 N-h) \Phi \theta_{3}^{n-16} \Delta_{8}^{2}\right) \quad \text { if } P \in \mathcal{H}^{(2)}\left(\mathbb{R}^{n}\right),
$$

where $c$ is a nonzero linear form on $\mathcal{H}^{(2)}\left(\mathbb{R}^{n}\right)$.

(iii) If $p \geqslant 1$ and if $L_{2}$ is not strongly eutactic, then

$$
\Theta_{\Lambda, P}=c_{1}(P) \Phi \theta_{3}^{n-8} \Delta_{8}+c_{2}(P) \Phi \theta_{3}^{n-16} \Delta_{8}^{2} \quad \text { if } P \in \mathcal{H}^{(2)}\left(\mathbb{R}^{n}\right),
$$

where $c_{1}$ and $c_{2}$ are linearly independent linear forms on $\mathcal{H}^{(2)}\left(\mathbb{R}^{n}\right)$.

We have checked numerically that the shells of norm at most 1200 of these lattices and of their shadows are not 3 -spherical designs.

\section{Appendix: The cubic lattices of rank 4 and 7}

The aim of this section is to prove the following result, without the use of modular forms:

\section{THEOREM.}

(i) Let $\mathbf{Z}^{4}=\left\{x=\left(x_{1}, \ldots, x_{4}\right) \in \mathbb{R}^{4} \mid x_{i} \in \mathbb{Z}\right\}$ be the cubic lattice of rank 4 , and let $m$ be an even positive integer. Then the shell $\left(\mathbf{Z}^{4}\right)_{m}$ is a 5 -design.

(ii) Let $\mathbf{Z}^{7}=\left\{x=\left(x_{1}, \ldots, x_{7}\right) \in \mathbb{R}^{7} \mid x_{i} \in \mathbb{Z}\right\}$ be the cubic lattice of rank 7 , and let $m$ be a positive integer of the form $m=4^{a}(8 b+3), a, b \geqslant 0$. Then the shell $\left(\mathbf{Z}^{7}\right)_{m}$ is a 5-design.

We begin with the proof of Claim (i). Let $C \subseteq(\mathbb{Z} / 2 \mathbb{Z})^{4}$ be the even weight code of length 4 , which is defined by

$$
c=\left(c_{1}, c_{2}, c_{3}, c_{4}\right) \in C \Longleftrightarrow c_{1}+c_{2}+c_{3}+c_{4}=0 \in \mathbb{Z} / 2 \mathbb{Z} .
$$

For $y=\left(y_{1}, \ldots, y_{4}\right) \in \mathbf{Z}^{4}$ we write $\bar{y} \in(\mathbb{Z} / 2 \mathbb{Z})^{4}$ its class modulo $2 \mathbf{Z}^{4}$. Let $\Lambda$ be the sublattice of $\mathbf{Z}^{4}$ consisting of elements $x \in \mathbf{Z}^{4}$ such that $\bar{x} \in C$. It is a sublattice of index 2 , which is equivalent to the root lattice of $\mathbf{D}_{4}$.

All shells of $\Lambda$ are 5-designs. This follows from the fact that $\Lambda$ is invariant under the Weyl group $\mathrm{W}\left(\mathbf{F}_{4}\right)$, and there is no nonconstant harmonic polynomial of degree at most 5 that is invariant under the action of $\mathrm{W}\left(\mathbf{F}_{4}\right)$ (GoeSei79, Thm. 6.1], GoeSei81 Thm. 3.12]; see also [HarPac04a, Sect. 4]).

Finally, for $m$ an even positive integer, we have $\left(\mathbf{Z}^{4}\right)_{m}=\Lambda_{m}$; therefore $\left(\mathbf{Z}^{4}\right)_{m}$ is a spherical 5-design. This proves Claim (i) of the Theorem.

Let us now show Claim (ii). Let $H \subseteq(\mathbb{Z} / 2 \mathbb{Z})^{7}$ be the Hamming code of length 7 . Recall that it is a linear code of minimal Hamming distance 3 , containing

- one codeword $\mathbf{0}:=(0,0,0,0,0,0,0)$ of weight 0 ;

- seven codewords of weight 3 ;

- seven codewords of weight 4 ;

- one codeword $\mathbf{1}:=(1,1,1,1,1,1,1)$ of weight 7 . 
The set $S$ of codewords of weight 3 forms a Steiner system $\mathrm{S}(2,3,7)$, and the set of codewords of weight 4 is $S+\mathbf{1}$. See for example [Ebel94, Sect. 1.2, p. 7].

Let $\Lambda$ be the sublattice of $\mathbf{Z}^{7}$ consisting of elements $x \in \mathbf{Z}^{7}$ such that $\bar{x} \in H$. It is a sublattice of index 8 , which is equivalent to $\sqrt{2} \mathbf{E}_{7}^{\sharp}$, the rescaled weight lattice of $\mathbf{E}_{7}$.

As in the previous case, all shells of $\Lambda$ are 5 -designs, because $\Lambda$ is invariant under the Weyl group $\mathrm{W}\left(\mathbf{E}_{7}\right)$, and there is no harmonic polynomial of degree at most 5 that is invariant under the action of $\mathrm{W}\left(\mathbf{E}_{7}\right)$.

But contrarily to the previous case, no shell of $\mathbf{Z}^{7}$ is equal to any shell of $\Lambda$. Therefore, we need to look at the effect of the action of some finite subgroup of the orthogonal group of rank 7 on the shells of $\Lambda$.

We recall first what is a weighted spherical design.

46. Definition. A weighted spherical t-design or spherical cubature formula of strength $t$ is the data consisting of a nonempty finite subset $X$ of $\mathbb{S}_{m}^{n-1}$ and a positive function $w: X \rightarrow \mathbb{R}_{>0}, x \mapsto w_{x}$, such that $\sum_{x \in X} w_{x}=1$ and such that the condition

$$
\sum_{x \in X} w_{x} P(x)=0, \quad \forall P \in \mathcal{H}^{(j)}\left(\mathbb{R}^{n}\right)
$$

holds for every integer $j$ with $1 \leqslant j \leqslant t$.

(For more on cubature formulae on spheres, see for example HarPac04b.)

Occasionaly, we allow the weight function $w: X \rightarrow \mathbb{R}$ to take the value zero. A spherical design is a weighted spherical design with constant weight function $w_{x}=1 /|X|$.

47. Lemma. Let $X \subseteq \mathbb{S}_{m}^{n-1}$ be a spherical $t$-design and let $G$ be a finite subgroup of the orthogonal group $\mathrm{O}(n)$. Then $G X$ is a weighted spherical t-design for the weight function

$$
w_{y}=\frac{|\{\sigma \in G \mid \sigma y \in X\}|}{|G||X|}, \quad y \in G X
$$

Proof. For $P \in \mathcal{H}^{(j)}\left(\mathbb{R}^{n}\right), 1 \leqslant j \leqslant t$, we have

$$
\sum_{y \in G X} w_{y} P(y)=\frac{1}{|G||X|} \sum_{\substack{x \in X \\ \sigma \in G}} P(\sigma x)=\frac{1}{|G||X|} \sum_{\sigma \in G}\left(\sum_{x \in X}(P \circ \sigma)(x)\right) .
$$

The last term is zero, because $P \circ \sigma \in \mathcal{H}^{(j)}\left(\mathbb{R}^{n}\right)$ and $X$ is a spherical $t$-design.

We apply the previous Lemma to the subgroup $G=\operatorname{Aut}\left(\mathbf{Z}^{7}\right) \simeq(\mathbb{Z} / 2 \mathbb{Z})^{7} \rtimes \mathfrak{S}_{7}$ of $\mathrm{O}(7)$, which consists of transformations of the form

$$
\left(x_{1}, \ldots, x_{7}\right) \mapsto\left(\epsilon_{1} x_{\pi(1)}, \ldots, \epsilon_{7} x_{\pi(7)}\right)
$$

where $\epsilon_{i} \in\{ \pm 1\}$ and $\pi$ is a permutation of the set $\{1,2, \ldots, 7\}$.

Let $c \in\{0,1, \ldots, 7\}$, and let $m$ be a positive integer such that $m \equiv c \bmod 8$. For $y=\left(y_{1}, \ldots, y_{7}\right) \in\left(\mathbf{Z}^{7}\right)_{m}$, let $W(y)$ be the weight of $\bar{y} \in(\mathbb{Z} / 2 \mathbb{Z})^{7}$, that is the number of coordinates $i$ such that $y_{i}$ is odd. (Caution: the word "weight" has two different meanings in this Appendix.) The condition $y_{1}^{2}+\cdots+y_{7}^{2} \equiv c \bmod 8$ implies that $W(y)$ takes the values indicated in the following table:

\begin{tabular}{c|cc|c}
$c$ & $W(y)$ & $c$ & $W(y)$ \\
\hline 0 & 0,4 & 4 & 0,4 \\
1 & 1,5 & 5 & 1,5 \\
2 & 2,6 & 6 & 2,6 \\
3 & 3 & 7 & 3,7
\end{tabular}


Now, the quantity

$$
\lambda(y):=\frac{\left|\left\{\sigma \in G \mid \sigma y \in \Lambda_{m}\right\}\right|}{|G|}, \quad \text { for } y \in\left(\mathbb{Z}^{n}\right)_{m}
$$

depends only of $W(y)$; it is given by the following table:

\begin{tabular}{c|c}
$W(y)$ & $\lambda(y)$ \\
\hline 0 & 1 \\
1 & 0 \\
2 & 0 \\
3 & $1 / 5$
\end{tabular}

\begin{tabular}{c|c}
$W(y)$ & $\lambda(y)$ \\
\hline 4 & $1 / 5$ \\
5 & 0 \\
6 & 0 \\
7 & 1
\end{tabular}

Applying Lemma 47 we find that

- for $m \equiv 0 \bmod 8$ and $m \equiv 4 \bmod 8$, the shell $\left(\mathbf{Z}^{7}\right)_{m}$ is a weighted spherical 5 -design for the weight function

$$
w_{y}=\frac{1}{\left|\Lambda_{m}\right|} \text { if } W(y)=0 \text {, and } w_{y}=\frac{1}{5\left|\Lambda_{m}\right|} \text { if } W(y)=4 ;
$$

- for $m \equiv 3 \bmod 8$, the shell $\left(\mathbf{Z}^{7}\right)_{m}$ is a spherical 5-design;

- for $m \equiv 7 \bmod 8$, the shell $\left(\mathbf{Z}^{7}\right)_{m}$ is a weighted spherical 5-design for the weight function

$$
w_{y}=\frac{1}{\left|\Lambda_{m}\right|} \text { if } W(y)=7 \text {, and } w_{y}=\frac{1}{5\left|\Lambda_{m}\right|} \text { if } W(y)=3 .
$$

In order to achieve the proof of Theorem 45 Claim (ii), it remains to show the following statement:

Let $m$ be a positive integer. If $\left(\mathbf{Z}^{7}\right)_{m}$ is a 5 -design, then $\left(\mathbf{Z}^{7}\right)_{4 m}$ is also a 5-design.

The proof is the following. We write $\left(\mathbf{Z}^{7}\right)_{4 m}$ as

$$
\left(\mathbf{Z}^{7}\right)_{4 m}=2\left(\mathbf{Z}^{7}\right)_{m} \sqcup Q
$$

where $2\left(\mathbf{Z}^{7}\right)_{m}$ is the shell of norm $m$ rescaled by a factor 2 , and $Q$ contains the elements $y \in\left(\mathbf{Z}^{7}\right)_{4 m}$ such that $W(y)=4$. We have shown that $\left(\mathbf{Z}^{7}\right)_{4 m}$ is a weighted 5 -design for the weight function

$$
w_{y}=\frac{1}{\left|\Lambda_{m}\right|} \text { if } y \in 2\left(\mathbf{Z}^{7}\right)_{m} \text {, and } w_{y}=\frac{1}{5\left|\Lambda_{m}\right|} \text { if } y \in Q .
$$

But since $\left(\mathbf{Z}^{7}\right)_{m}$ is a 5-design by hypothesis, then $\left(\mathbf{Z}^{7}\right)_{4 m}$ is a weighted 5-design for the weight function

$$
\widetilde{w}_{y}=\frac{1}{\left|\left(\mathbf{Z}^{7}\right)_{m}\right|} \text { if } y \in 2\left(\mathbf{Z}^{7}\right)_{m}=0 \text {, and } \widetilde{w}_{y}=0 \text { if } y \in Q .
$$

It is evident that, if $X$ is a weighted $t$-design for two weight functions $w$ and $\widetilde{w}$, then it is a weighted $t$-design for every convex linear combination of $w$ and $\widetilde{w}$.

In particular, since, in our case, there is a suitable convex linear combination of $w$ and $\widetilde{w}$ which is constant on $\left(\mathbf{Z}^{7}\right)_{4 m}$, the shell $\left(\mathbf{Z}^{7}\right)_{4 m}$ is indeed a 5-design.

Remark that our proof also shows that the shells of $\mathbf{Z}^{7}$ of norm $m \equiv 7 \bmod 8$ and those of norm $m \equiv 0 \bmod 4$ are weighted spherical 5-designs, although they are not spherical 5-designs in general.

Acknowledgements. The author thanks Boris Venkov, who was at the origin of this work, and who introduced the author to the use of modular forms in connection with lattices. Pierre DE LA HARPE also contributed to this work.

The author acknowledges support from the Swiss National Science Foundation. 


\section{References}

[Bach97] R. Bacher, Tables de réseaux entiers construits comme $k$-voisins de $\mathbb{Z}^{n}$, J. Théor. Nombres Bordeaux 9 (2) (1997) 479-497.

[BacVen01] Ch. Bachoc, B.B. Venkov, Modular forms, lattices and spherical designs, chap. 2 of MartV01

[BekHar02] B. Bekka, P. de la Harpe, Irreducibility of unitary group representations and reproducing kernels Hilbert spaces, Expo. Math. 21 (2) (2003) 115-149.

[Borc84] R.E. Borcherds, The Leech lattice and other latices, Ph.D. dissertation, Univ. of Cambridge 1984.

[BorcCS] R.E. Borcherds, The 24-dimensional odd unimodular lattices, Chap. 17 of ConSlo99.

[Bour81] N. Bourbaki, Groupes et algèbres de Lie, Masson (Paris) 1981.

[ConSlo99] J.H. Conway, N.J.A. Sloane, Sphere Packings, Lattices and Groups, third edition, Springer (New York) 1999.

[DeGoSe77] P. Delsarte, J.-M. Goethals, J.J. Seidel, Spherical codes and designs, Geometriae Dedicata 6 (1977) 363-388.

[Ebel94] W. Ebeling, Lattices and codes, a course partially based on lectures by F. Hirzenbruch, Vieweg (Braunschweig) 1994. Second revised edition: 2002.

[Elki95a] N.D. Elkies, A characterization of the $\mathbf{Z}^{n}$-lattice, Math. Res. Lett. 2 (3) (1995) 321-326.

[Elki95b] N.D. Elkies, Lattices and codes with long shadows, Math. Res. Lett. 2 (5) (1995) 643-645.

[GoeSei79] J.-M. Goethals, J.J. Seidel, Spherical Designs, Proc. Sympos. Pure Math., XXXIV, 255-272, Amer. Math. Soc. (Providence R.I.) 1979.

[GoeSei81] J.-M. Goethals, J.J. Seidel, Cubature formulae, polytopes, and spherical designs, in: The geometric vein, the Coxeter festschrift, Springer (New York, Berlin) (1981) 203-218.

[HarPac04a] P. de la Harpe, C. Pache, Spherical designs and finite group representations (some results of E. Bannai), European J. Combin. 25 (2) (2004) 213-227.

[HarPac04b] P. de la Harpe, C. Pache, Cubature formulas, geometrical designs, reproducing kernels, and Markov operators, in preparation.

[MartV01] J. Martinet ed., Réseaux euclidiens, designs sphériques et formes modulaires, Autour des travaux de B. Venkov, L'Enseignement Mathématique, monographie no 37 (Genève) 2001.

[NebVen00] G. Nebe, B.B. Venkov, The strongly perfect lattices of dimension 10, J. Théor. Nombres Bordeaux 12 (2) (2000) 503-518.

[Rank77] R.A. Rankin, Modular forms and functions, Cambridge University Press, 1977. 
[SchSch99] R. Scharlau, R. Schulze-Pillot, Extremal lattices, in: Algorithmic algebra and number theory (Heidelberg, 1997), Springer (Berlin) (1999) 139-170.

[Serr85] J.-P. Serre, Sur la lacunarité des puissances de $\eta$, Glasgow Math. J. 27 (1985) 203-221 [= Euvres, Volume IV, 66-84, see also 640].

[Venk78] B.B. Venkov The classification of integral even unimodular 24-dimensional quadratic forms, Trudy Mat. Inst. Steklova 148 (1978) 65-76 $=$ Proc. Steklov Inst. Math. $\mathrm{n}^{\mathrm{o}} 4$ (1980) 63-74

$\simeq$ Even Unimodular 24-Dimensional Lattices, Chap. 18 of ConSlo99.

[Venk84] B.B. Venkov, Even unimodular extremal lattices, Tr. Mat. Inst. Steklova 165 (1984) 43-48 (Russian) = Proc. Steklov Inst. Math. 165 (3) (1985) 47-52.

[VenMar01] B.B. Venkov (notes by J. Martinet), Réseaux et designs sphériques, Chap 1 of MartV01.

[Vile68] N.Ya. Vilenkin, Специальные функиии и теория представлений zpynn, second edition, Nauka (Moscow) 1991

= Special Functions and the Theory of Group Representations, Transl. Math. Monographs 22, Amer. Math. Soc. (Providence R.I.) 1968

$=$ Fonctions spéciales et théorie de la représentation des groupes, Dunod (Paris) 1969.

[Voro08] G. Voronoi, Nouvelles applications des paramètres continus à la théorie des formes quadratiques: 1. Sur quelques propriétés des formes quadratiques positives parfaites, J. reine angew. Math. 133 (1908) 97178. 